\title{
Installation Summaries from the 1996 Survey of Threatened and Endangered Species on Army Lands
}

by

Eric R. Schreiber, Rachel A. Shaw, Alison Hill, and Mary A. Reed

In November of 1996, the Army conducted a survey of the Threatened and Endangered (T\&E) species residing on and contiguous to its lands. This report, intended for installation use, provides quick access to basic information from the survey on the Federally Threatened, Endangered, Proposed, and Candidate (TEPC) species that occur on Army lands. In addition to a summary of Army-wide information, data is also provided at the installation level and the species level. Installation level data provides information on all species which occur on each installation, while species level data lists all installations on which each species occurs. Corresponding information on Federal status, critical habitat, and endangered species management plans (ESMPs) are provided for each species, in addition to points-of-contact for each installation. 

SF 298 


\section{Foreword}

This study was conducted for the U.S. Army Environmental Center (AEC) and the Office of the Directorate of Environmental Programs (ODEP), Assistant Chief of Staff for Installation Management (ACSI M) under Military I nterdepartmental Purchase Request (MIPR) No. 3176; Work Unit ND6, "1996 TES Survey, Phase 1: Survey Development and Administration," and MIPR No. 2357; Work Unit KG7, "1996 TES Survey, Phase 2: Analysis and Reporting." The technical monitor was Scott Belfit, SFIM-AEC-ECM.

The work was performed by the Natural Resource Assessment and Managment Division (LL-N) of the Land Management Laboratory (LL), U.S. Army Construction Engineering Research Laboratories (USACERL). Mary A. Reed, U.S. Army Environmental Center, assisted in the technical review. The USACERL principal investigator was Dr. Alison Hill. Robert M. Lacey is Acting Chief, CECER-LL-N; Dr. William D. Severinghaus is Operations Chief, CECER-LL. The USACERL technical editor was Gloria J . Wienke, Technical Resources Center.

COL J ames A. Walter is Commander and Dr. Michael J. O'Connor is Director of USACERL. 


\section{Contents}

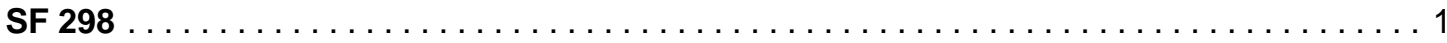

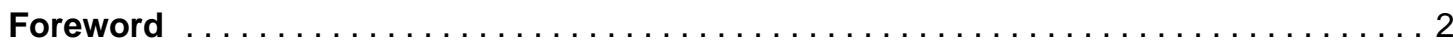

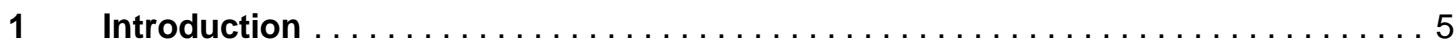

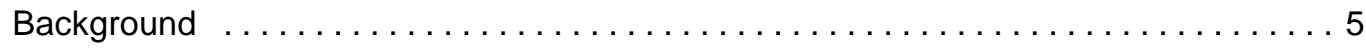

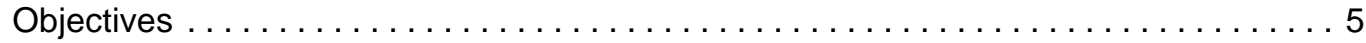

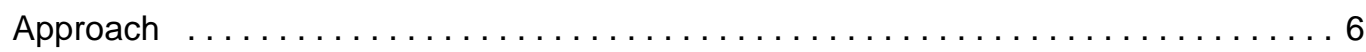

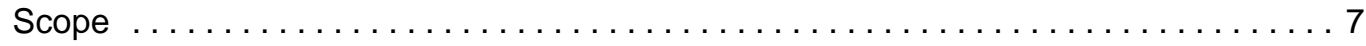

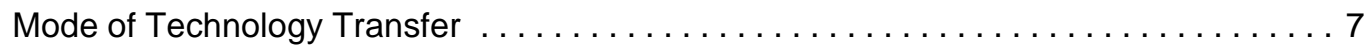

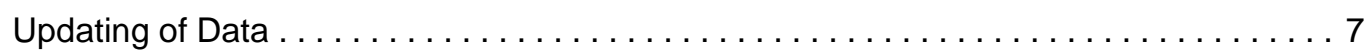

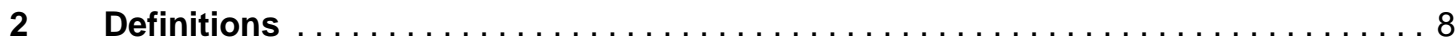

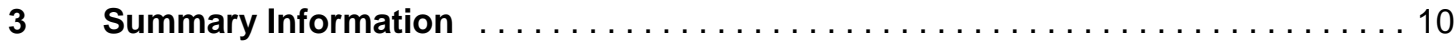

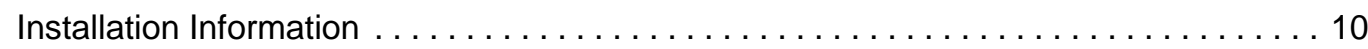

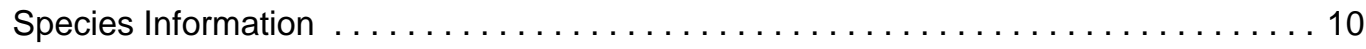

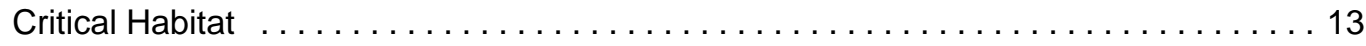

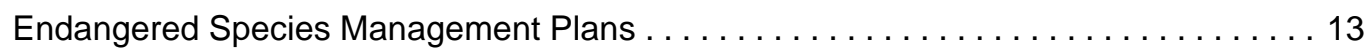

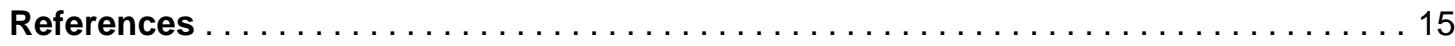

Appendix A: Onsite and Continguous TEPC Species, by Installation, with Corresponding

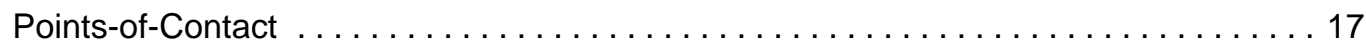

Appendix B: Critical Habitat and ESMP Status for Onsite and Contiguous TEPC Species, with

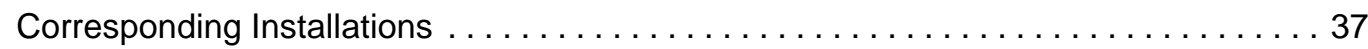

\section{Distribution}




\section{Introduction}

\section{Background}

The Army is required to protect state and Federally listed Threatened and Endangered (T\&E) species on its lands (Army Regulation [AR] 420-74 1989). This requirement directly affects land use and management. The presence of T\&E species can: (1) limit land uses, induding training activities, (2) result in direct conflicts with the military mission, (3) require added financial and personnel resources to take affirmative action on behalf of listed species, and (4) involve state and Federal wildlife agencies in Army land use decision-making (Tazik, Sherman, and Courson 1991).

Compared with other Federal land management agencies, the military has a disproportionately large number of T\&E species on its lands (Flather et al. 1994). In some instances, this has resulted in conflict between T\&E conservation requirements and the installation commander's ability to train soldiers and test weapons and equipment to assure readiness. The Army must be able to determine the extent of potential T\&E species problems in order to develop long-term management strategies while minimizing negative effects on training and testing. Because of the potential for conflict between $T \& E$ conservation and training/testing, the Office of the Directorate of Environmental Programs (ODEP) and the Army Environmental Center (AEC) are pursuing innovative ways to manage and monitor the number of T\&E species on Army lands. Army leadership is committed to developing and supporting a reliable and comprehensive source of T\&E species information to ensure compliance with the Endangered Species Act (ESA), Army policy, and training and testing readiness. Standardized annual T\&E species data collection will allow the Army to respond to mandated information requests, and track the progress of T\&E species management Army-wide (Shaw and Hill 1996).

\section{Objectives}

The objectives of this research were to:

- summarize available T\&E species information from the 1996 TES survey, 
- $\quad$ provide installations with a list of species having critical habitat and endangered species management plans, and

- $\quad$ provide points-of-contact for obtaining additional information.

\section{Approach}

The project consisted of two phases. Phase One was to develop a T\&E survey for installations. Phase Two was to administer the survey and conduct analysis and reporting.

Phase One: Development of the Survey Instrument. The 10-month development process required substantial collaboration with the T\&E Species Users Group, an advisory group consisting of members of ODEP, Army Major Commands (MACOMs), AEC, and the Office of the Deputy Chief of Staff for Operations and Plans (ODSCOP), and consultation with University of Illinois survey research experts. This phase required soliciting ideas and questions, facilitating discussions about the wording of the questions, coordinating the review of revisions, and pretesting by potential survey recipients.

Phase Two: Administration, Analysis, and Reporting. In November 1996, ODEP directed the MACOM Commanders to distribute the survey instructions and instrument, a glossary, a listing of species from the Federal Register, and a reference listing of species reported previously on installations. The packet was forwarded to installations, with a 45-day suspense. An accompanying memorandum suggested installation recipients assemble a team to fill out the survey. These key individuals might include personnel from the Directorate of Public Works (DPW) or equivalent, the Directorate of Plans and Training Mission (DPTM) or equivalent, and the J udge Advocate General ( $A G$ ) or compliance office that handle T\&E issues.

A complete survey package went to all Army installations with the potential for $T \& E$ species. MACOMs assisted with determining installations having the potential for T\&E species. MACOM information included acreage, known species occurrences, previous TES surveys, knowledge of the installation, etc. Installations were asked about Federally Threatened, Endangered, Proposed, and Candidate (TE PC) species known to reside on, or contiguous to, the installation. The survey consisted of approximately 50 questions. Questions asked for: (1) T\&E management and compliance concerns, (2) training and testing restrictions/impacts due to $T \& E$ species, and (3) points-of-contact. 


\section{Scope}

This report provides the 1996 overview of the T\&E species on Army installations, and includes 248 distinct TEPC species (523 occurrences) for 201 Army installations, with corresponding information on critical habitat and endangered species management plans (ESMPs).

\section{Mode of Technology Transfer}

Headquarters, Department of the Army (HQDA) and MACOMS were provided raw data resulting from this survey. This report provides installations with summary TES information for future reference.

\section{Updating of Data}

The data is current as of $\mathrm{J}$ anuary 10, 1997. Any incorrect species, misidentifications, changes in ESMP status, or new species not known at the time of the survey administration, will not be added, but will become part of the 1997 survey.

This report is the third of a series of three, with the intended audience being the Environmental and Training Managers at installations. The first report, titled The 1996 Survey of Threatened and Endangered Species on Army Lands: A Summary Report (Shaw, Schreiber, and Hill 1997), provides (HQDA) and MACOM summaries based on survey responses. The second report, titled Threatened and Endangered Species on Army Installations: A MACOM Report (Schreiber, Shaw, and Hill 1997), also provides HQDA and MACOM-level summaries; it includes installation profiles, specifying MACOM, county, point-of-contact, and a listing of all onsite and contiguous species by category, for each of the 201 installations responding to the survey. 


\section{Definitions}

Several acronyms and terms are used consistently throughout this document. These terms are defined below:

Distinct Species - The term distinct species is synonymous with the term unique species. For example, the bald eagle is considered as one distinct or unique species, regardless of the number of installations on which it occurs. Distinct species may be calculated Army-wide, or within individual MACOMs. In either case, a particular species is counted only once.

F ederal Status

E - Federally Endangered

$T$ - Federally Threatened

PE - Federally Proposed as Endangered

PT - Federally Proposed as Threatened

C - Candidate species for Federal listing

Installation - The term installation refers to active military installations, state National Guard Bureau (NGB) offices, NGB training sites, and regional support centers for the Army Reserve. In some cases,it was difficult to define "installation" data because some NGB state offices, for example, reported on a state-wide basis, while others reported for each training site in the state. Analyses in this report do not make a distinction between these types of locations.

Occurrence - An occurrence is a record of a species. A record refers to each time a species is recorded as being present on an installation and is synonymous with the term record in terms of the database. F or example, if a bald eagle occurs on five installations it is recorded as five occurrences.

S/A - Similarity of Appearance. Certain species that closely resemble T\&E species may be given the Federal status of the species they resemble. Similarity of appearance species are denoted with a $\mathrm{T}(\mathrm{S} / \mathrm{A})$ or $\mathrm{E}(\mathrm{S} / \mathrm{A})$. However, no distinction is made between $S / A$ species and other $T \& E$ species. 
Species Location

Both - Species known to occur both onsite and contigous to the installation.

Contiguous- Species known to occupy or otherwise use habitat adjacent to an installation, which may be affected by installation military activities and land management practices.

Onsite - Species that have been documented within the boundaries of the installation.

T\&E - The term T\&E indudes only Federally Threatened and Endangered species. These two statuses include S/A species.

TEP - The term TEP includes all Federally Threatened, Endangered, Proposed Threatened, and Proposed Endangered species on Army lands but does not include Candidate species.

TEPC - The term TEPC includes all Federally Threatened, Endangered, Proposed Threatened, Proposed Endangered, and Candidate species on Army lands. Subsequent analyses that use the term TEPC refer to the status of all species. 


\section{Summary Information}

\section{Installation Information}

A total of 201 installations responded to the 1996 T\&E species survey. Of those responding, 105 (52\%) installations have onsite TEPC species, 84 (42\%) installations have no TEPC species, and 12 (6\%) installations have strictly contiguous species. Of the 105 installations with onsite TEPC species, 100 have T\&E species.

The 201 installations include: 48 Army Materiel Command (AMC), 20 Forces Command (FORSCOM), 6 Military District of Washington (MDW), 4 Medical Command (MEDCOM), 3 Military Traffic Management Command (MTMC), 80 National Guard Bureau (NGB), 16 Training and Doctrine Command (TRADOC), 14 U.S. Army Reserve Command (USARC), 9 U.S. Army, Pacific (USARPAC), and 1 U.S. Military Academy (USMA).

Appendix A lists the $117^{1}$ installations with onsite or contiguous TEPC species, with corresponding information on scientific name, common name, Federal status, category, occurrence, critical habitat, and ESMP status for each species.

\section{Species Information}

A total of 248 distinct onsite and contiguous TEPC species (523 occurrences) were identified on 117 (58\%)of the installations surveyed. These 248 TEPC species indude: 147 onsite ${ }^{2}$ and contiguous species (265 occurrences), 54 onsite species (128 occurrences), and 47 contiguous species (130 occurrences) (see Table 1). Of the 201 distinct onsite TEPC species, 153 are T\&E.

\footnotetext{
1 This number includes 105 installations with onsite TEPC species and 12 installations with strictly contiguous TEPC species.

2 It should be noted that some species listed as onsite may also occur contiguous to the installation.
} 
Table 1. Installation and species summary information.

\begin{tabular}{|l|r|r|r|}
\hline & $\begin{array}{r}\text { Number of } \\
\text { installations }\end{array}$ & $\begin{array}{r}\text { Number of } \\
\text { distinct species }\end{array}$ & $\begin{array}{r}\text { Number of species } \\
\text { occurrences }\end{array}$ \\
\hline Totals & 201 & 248 & 523 \\
\hline Onsite TEPC species & 105 & 201 & 393 \\
\hline No TEPC species & 84 & 0 & 0 \\
\hline Strictly contiguous species & 12 & 47 & 130 \\
\hline Onsite T\&E species* & 100 & 153 & 430 \\
\hline * Onsite T\&E species are independent of the overall TEPC species numbers and cannot be added. \\
\hline
\end{tabular}

Of the 248 distinct species, $64 \%$ are Endangered, $14 \%$ are Threatened, $13 \%$ are Proposed Endangered, $8 \%$ are Candidates, and $1 \%$ are Proposed Threatened (F igure 1). When listed by Category, $54 \%$ of the 248 distinct species are plants, $15 \%$ are birds, $7 \%$ are snails, $5 \%$ are reptiles, fish, and mammals, $3 \%$ are clams and insects, $2 \%$ are amphibians, and $1 \%$ are crustaceans (Figure 2 ).

\section{Federal Status}

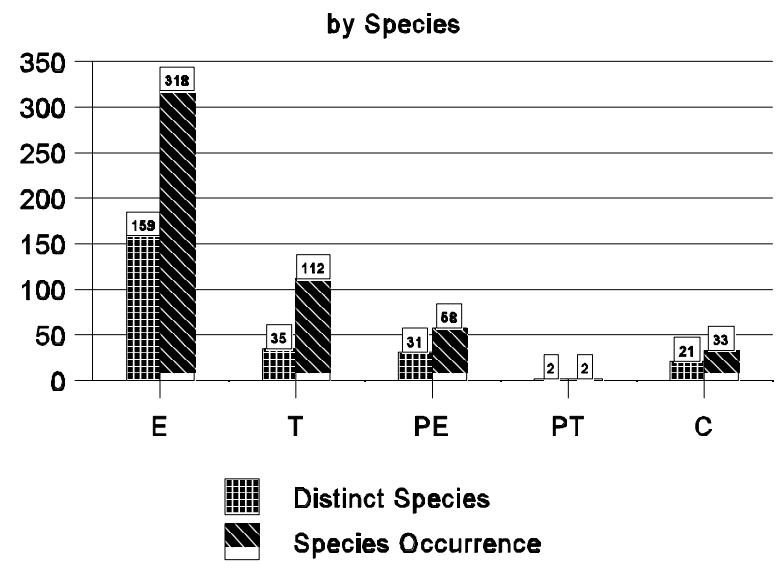

Figure 1. Federal status, by distinct species and species occurrence, for all species combined. 


\section{Distinct Species}

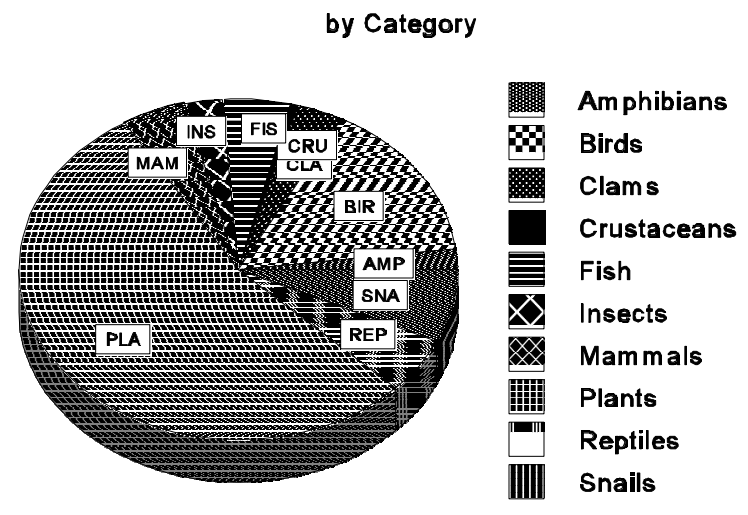

Figure 2. Distinct species, by category, for all species combined.

Of the 90 contiguous TEPC species, $67 \%$ are Endangered, $16 \%$ are Threatened, $9 \%$ are Candidates, $8 \%$ are Proposed Endangered, and 1\% are Proposed Threatened (Figure 3). When divided by category, 37\% of the contiguous species are plants, $24 \%$ are birds, $10 \%$ are fish, $8 \%$ are clams, $6 \%$ are mammals and reptiles, $4 \%$ are snails, $3 \%$ are insects, and $2 \%$ are amphibians (Figure 4 ).

\section{Federal Status}

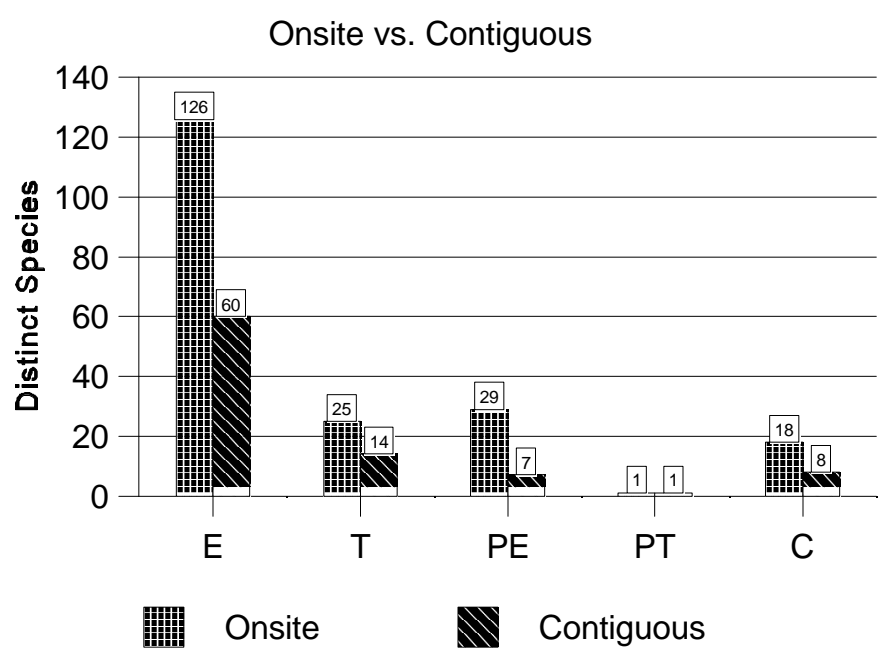

Figure 3. Distinct species, by Federal status, for onsite and contiguous species. 


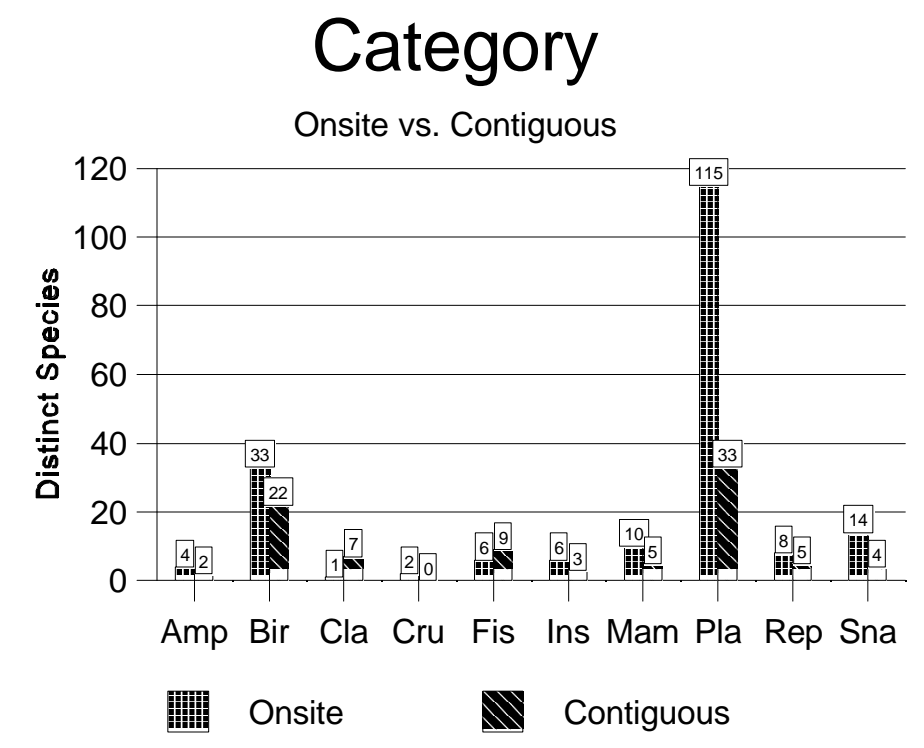

Figure 4. Distinct species, by category, for onsite and contiguous species.

Appendix B lists the 248 distinct onsite and contiguous TEPC species, with information on occurrence, critical habitat, and ESMP status for each of the corresponding installations.

\section{Critical Habitat}

Installations reported 21 distinct T\&E species (32 occurrences) as having critical habitat. However, when checked against the Federal Register, only 5 species (6 occurrences) were confirmed as having critical habitat. ${ }^{3}$ Confirmed species with critical habitat include the Mexican spotted owl (Camp Navajo and Fort Huachuca), northern spotted owl (Fort Lewis), palila (Pohakuloa), desert tortoise (Fort I rwin), and the Oregon silverspot butterfly (Camp Rilea).

\section{Endangered Species Management Plans}

Of the 393 onsite Federal TEPC species occurrences, only 371 were considered "eligible" to have an endangered species management plan (ESMP). The term "eligible" refers to F ederally Threatened, Endangered, and Proposed species (TEP), and assumes that Candidate species should not have an ESMP.

\footnotetext{
3 Unconfirmed records were changed in the database to reflect the status reported in the Federal Register.
} 
Of the 371 "eligible" onsite TEP species occurrences, 219 (59\%) have an ESMP that is currently under preparation or in process, 103 (28\%) have not yet had an ESMP initiated, 36 (10\%) have a completed ESMP, and 13 (4\%) had no response.

The Army also had 46 contiguous TEP species occurrences that were considered "eligible" to have an ESMP. Of these occurrences, 40 (87\%) have an ESMP that is currently under preparation or in process, and 6 (13\%) have a completed ESMP. 


\section{References}

\section{Cited}

16 U.S. Code (U.S.C.) 1536(a)(1); Army Regulation (AR) 420-74, Paragraph 6-2 Natural Resource - Land, Forest, and Wildl ife Management (Headquarters, Department of the Army [HQDA], 25 February 1986); Federal Register (FR) 54:7539-7543, 265.7(B)(3)(b) [22 Feb 1989].

Flather, C. H., L.H.J oyce, and C.A. Bloomgarden. Species endangerment patterns in the United States. Gen. Tech. Rep. RM-241. Fort Collins, CO: U.S. Department of Agriculture, Forest Service, Rocky Mountain Forest and Range Experiment Station, 1994. 42pp.

Schreiber, E.R., R.A. Shaw, A. Hill, and M.A. Reed, Threatened and Endangered Species on Army Installations: A MACOM Report, Technical Report [TR] 98/18 (U.S. Army Construction Engineering Research Laboratory [USACERL], December 1997).

Shaw, R.A., and A. Hill, Army-wide Threatened and Endangered Species Survey, USACERL Technical Note 96-101 (U.S. Army Construction Engineering Research Laboratory, September 1996).

Shaw, R.A., E.R. Schreiber, and A. Hill, The 1996 Survey of Threatened and Endangered Species on Army Lands: A Summary Report, Technical Report [TR] 98/17 (USACERL, December 1997).

Tazik, D.J ., R.A. Sherman, and J .A. Courson, Annotated Directory of Threatened and Endangered Wildlife on Selected U.S. Army Installations E ast of the Mississippi River, Technical Report [TR] N-91/26 (USACERL, May 1991). 


\section{Appendix A: Onsite and Contiguous TEPC Species, by Installation, with Corresponding Points-of-Contact}




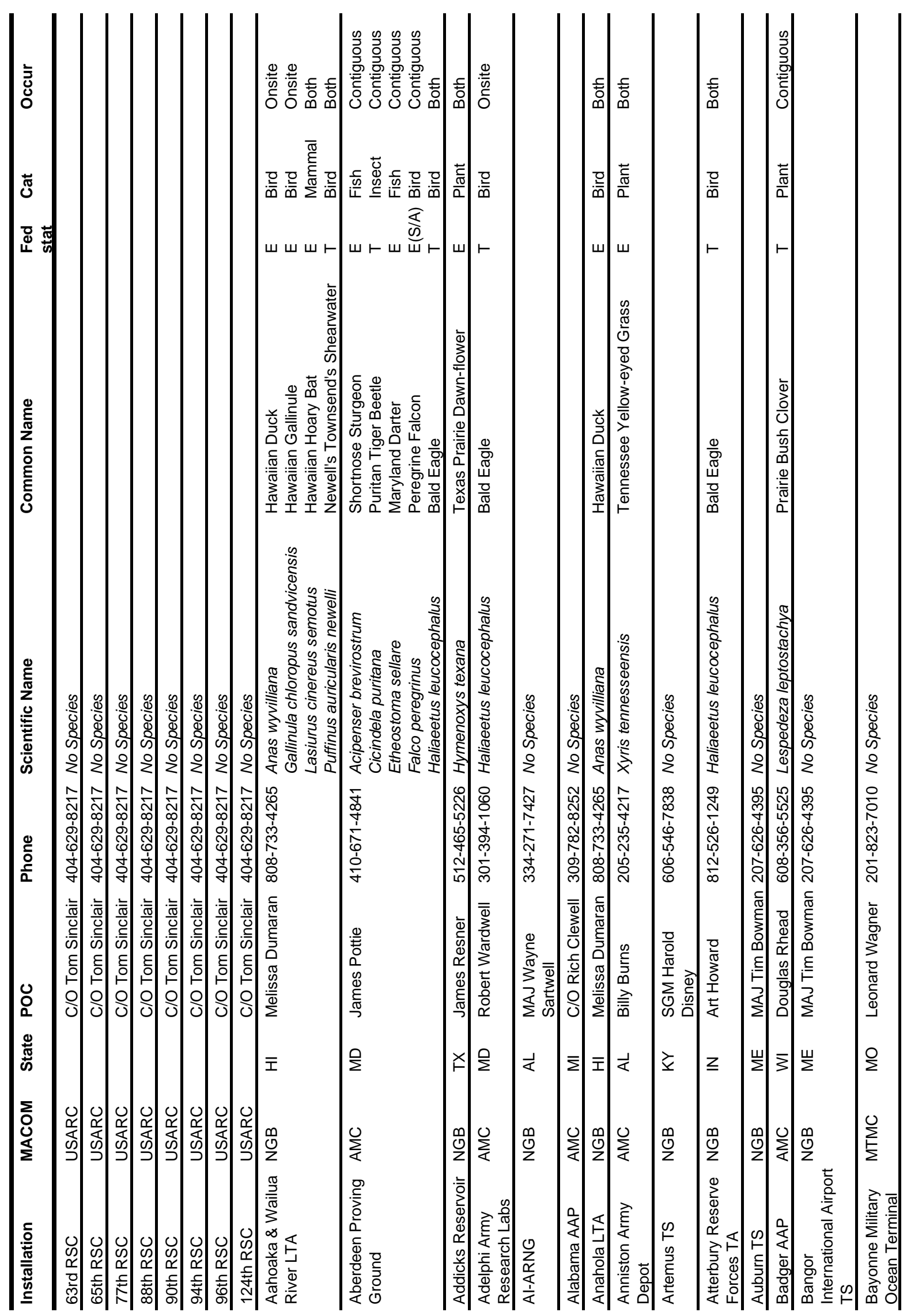




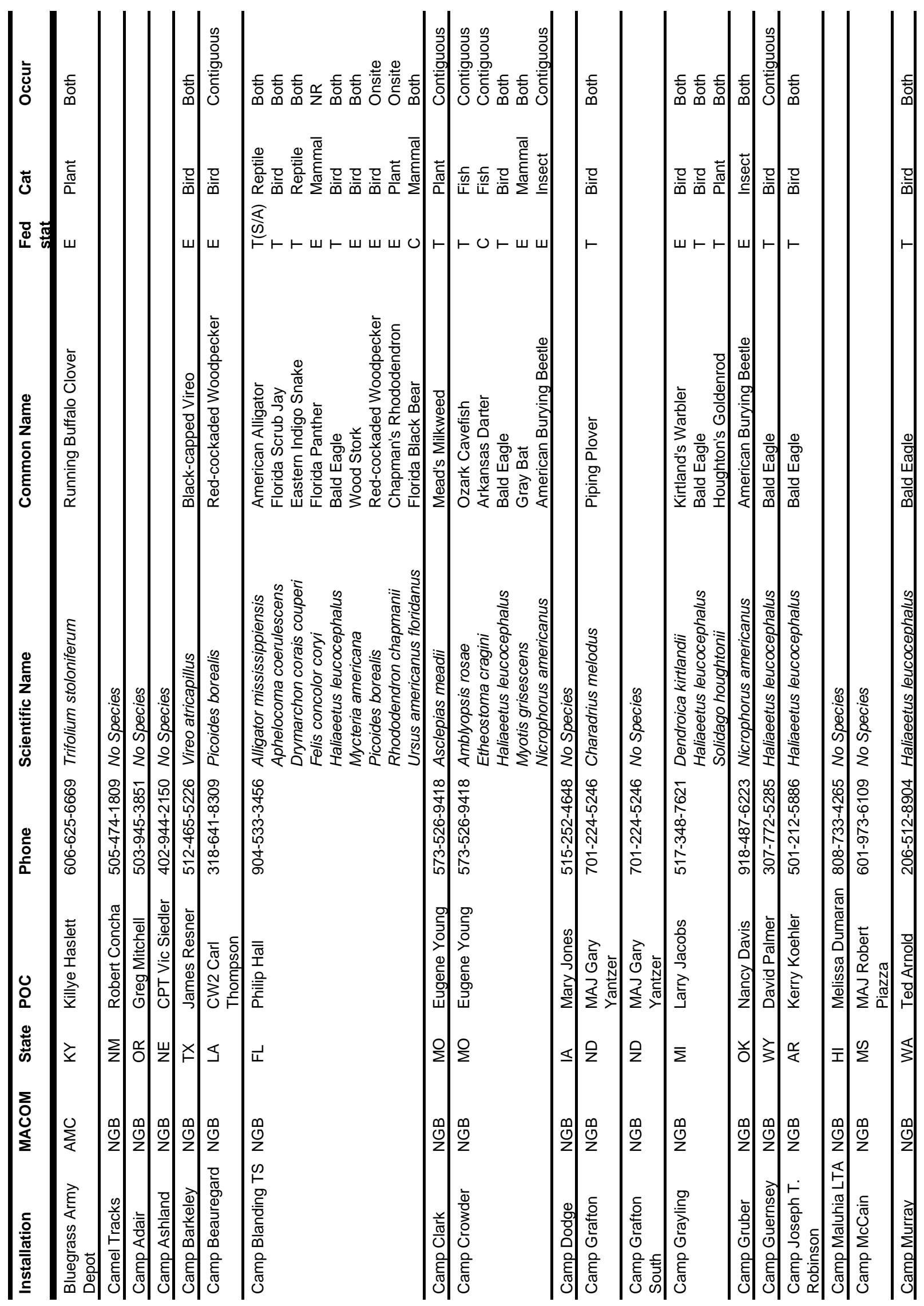




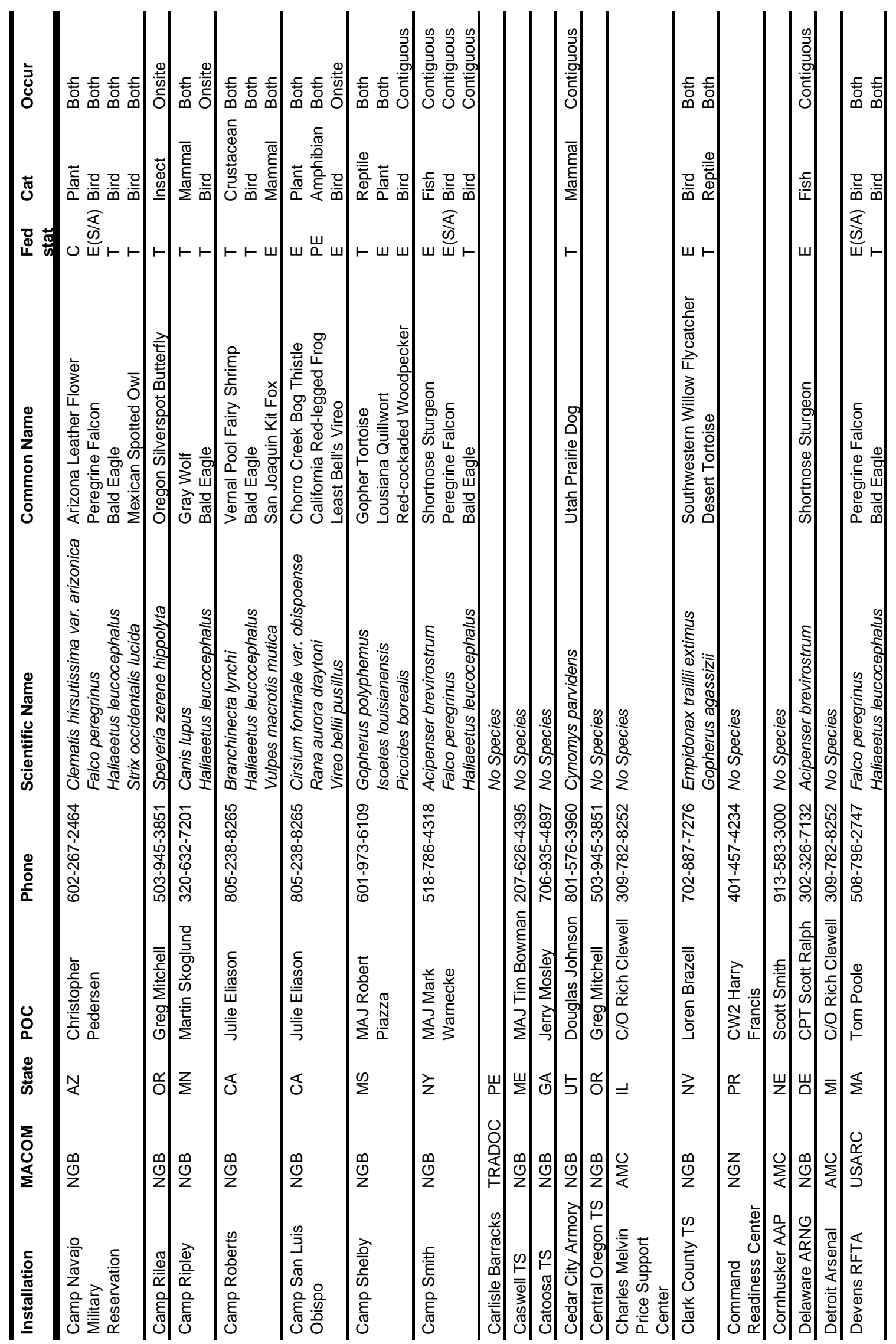




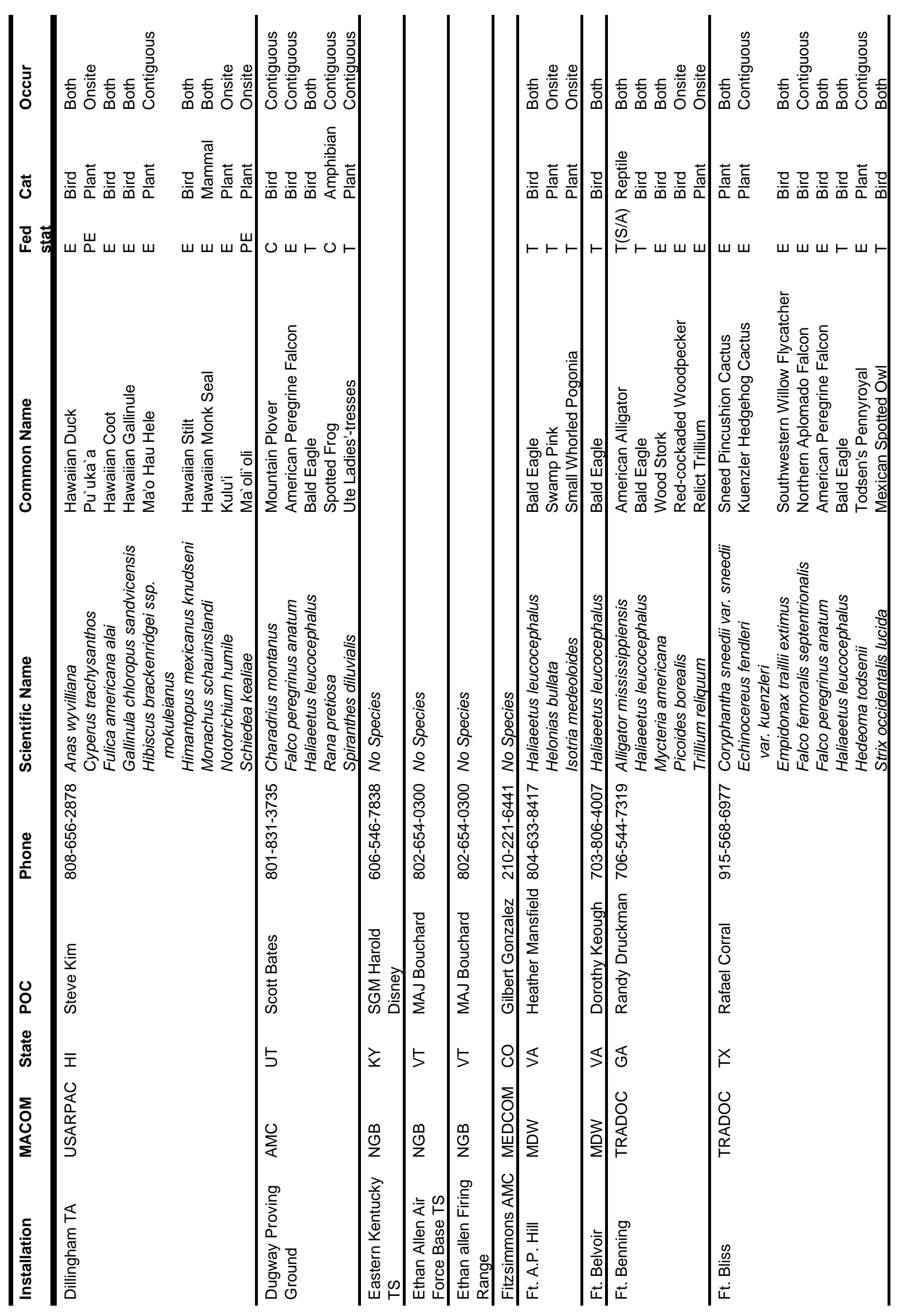




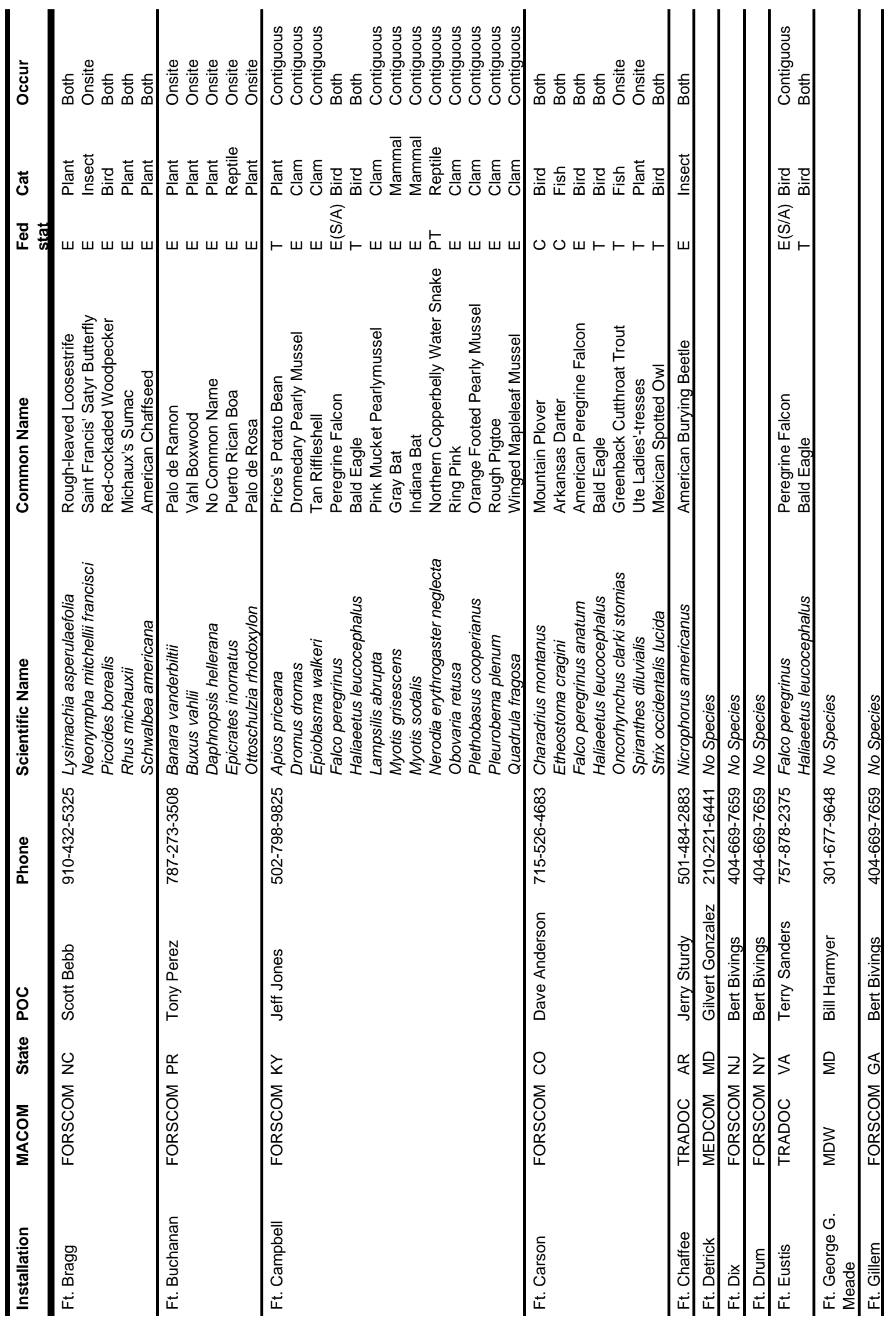




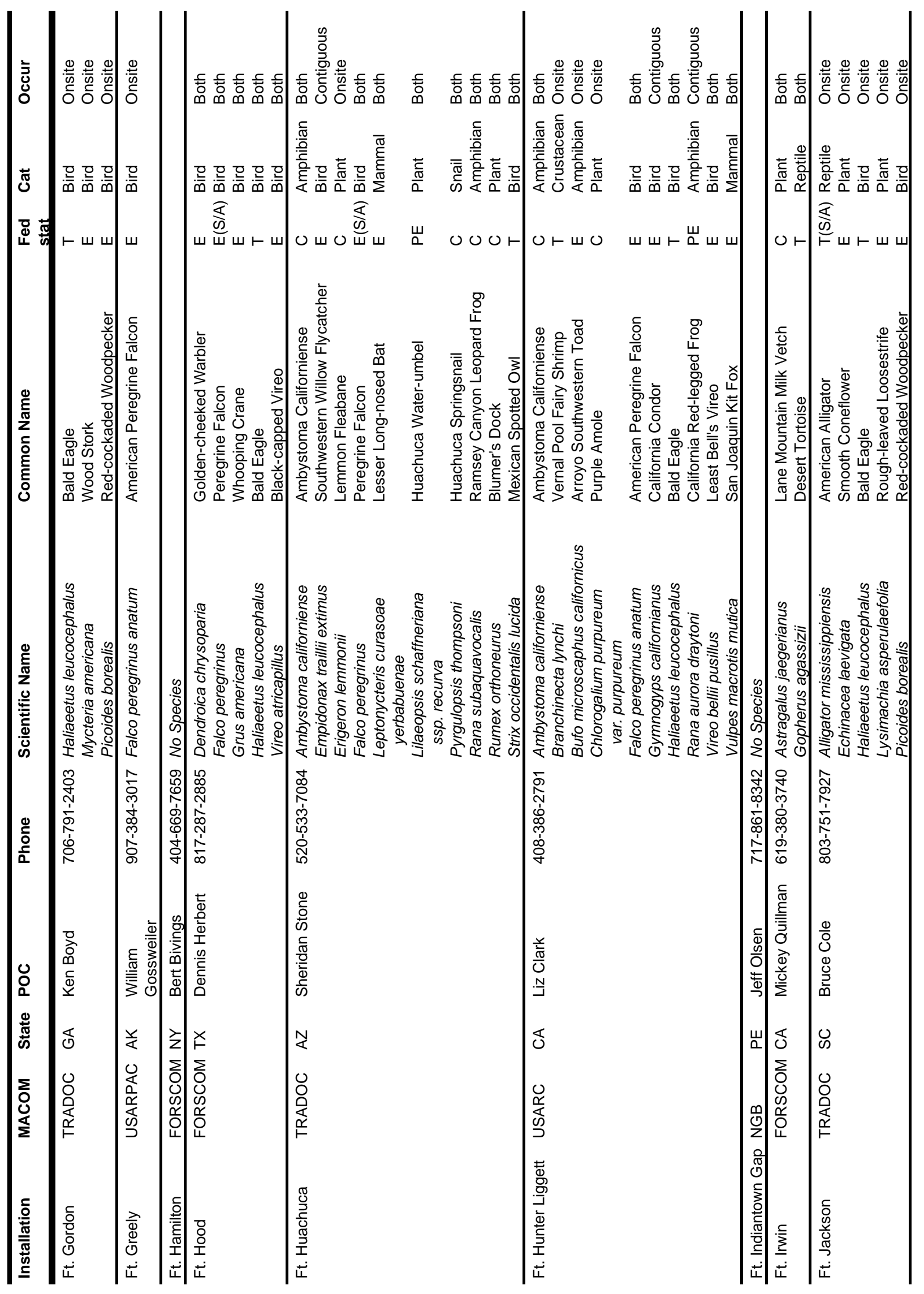




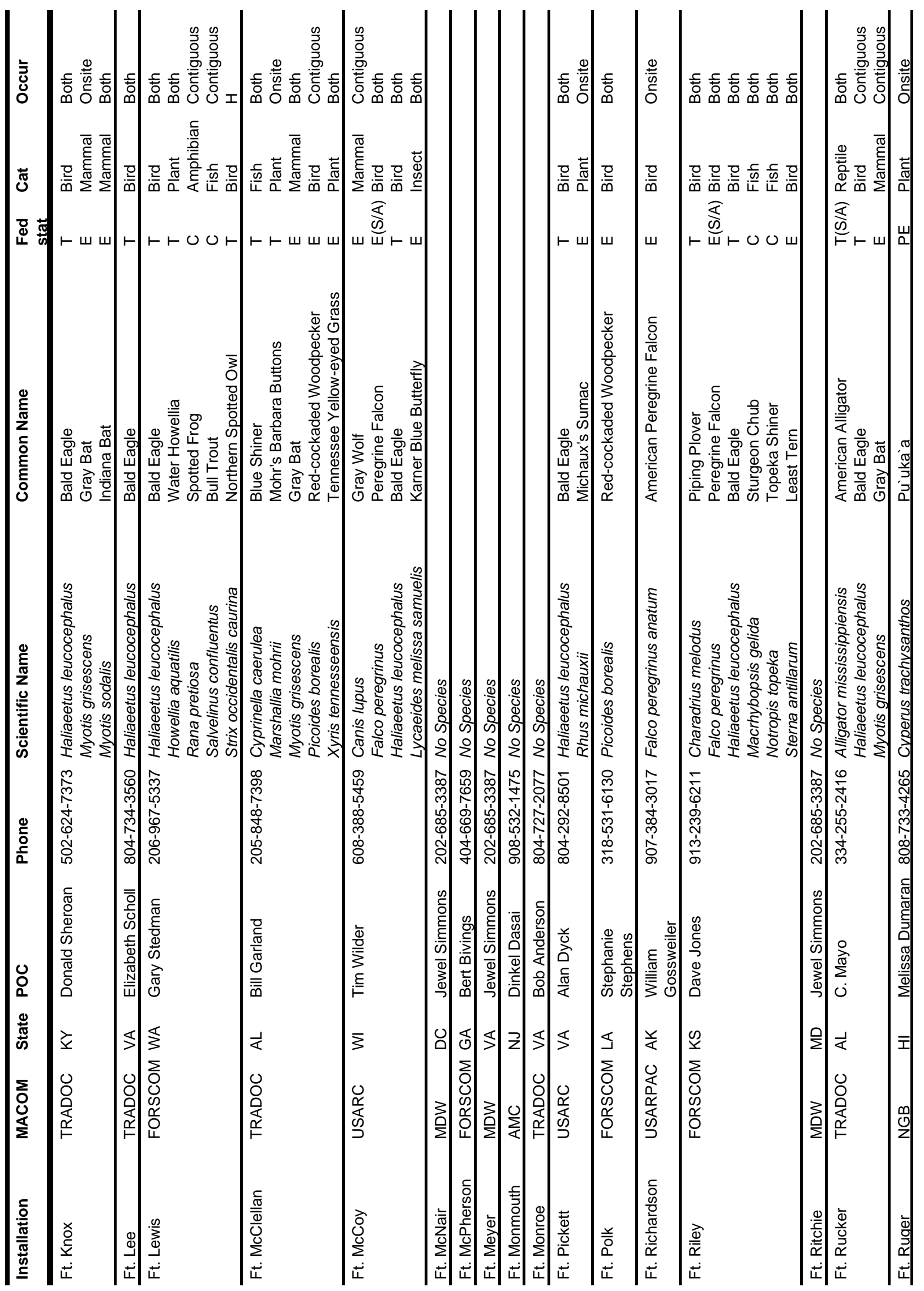




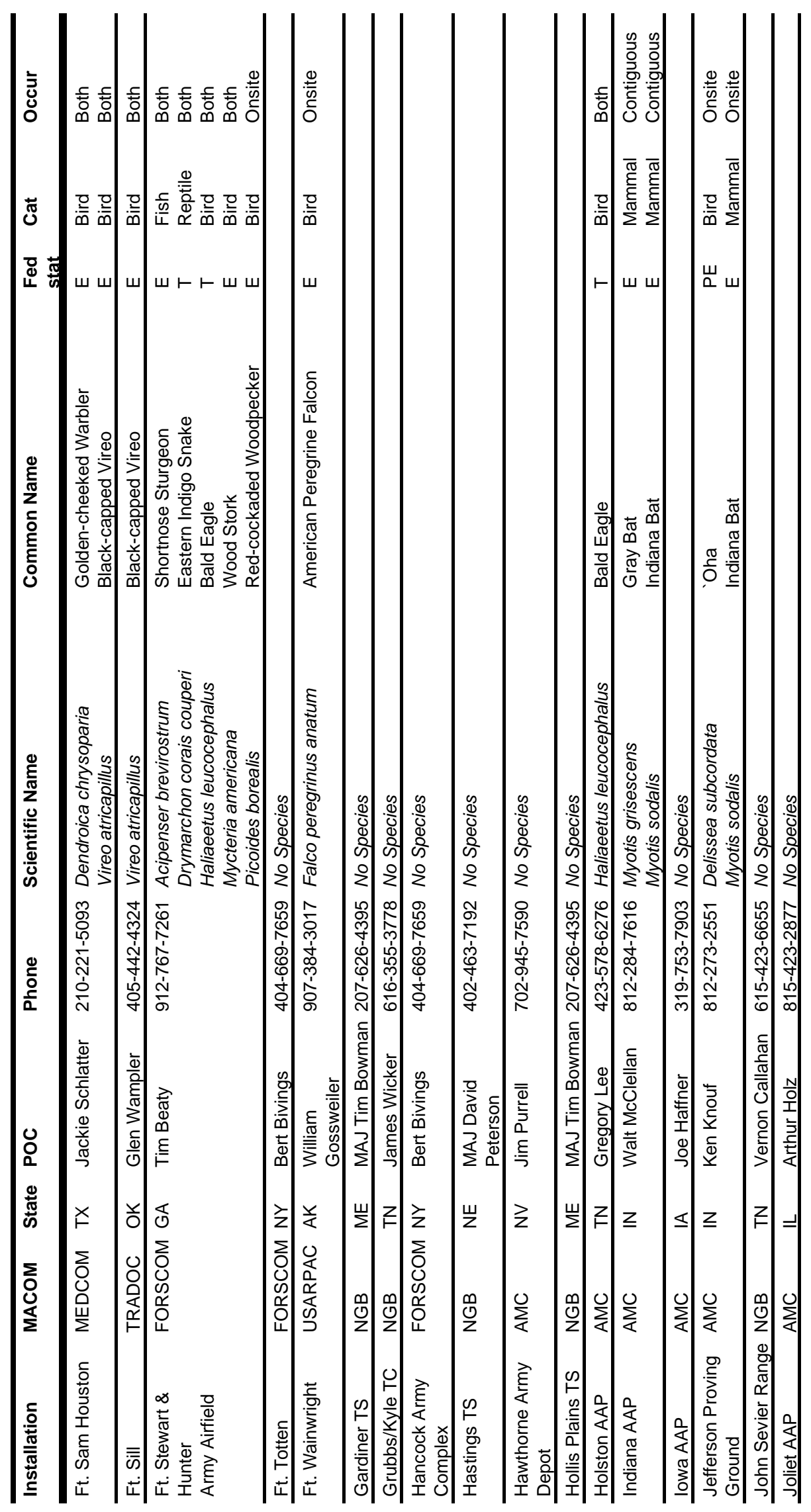




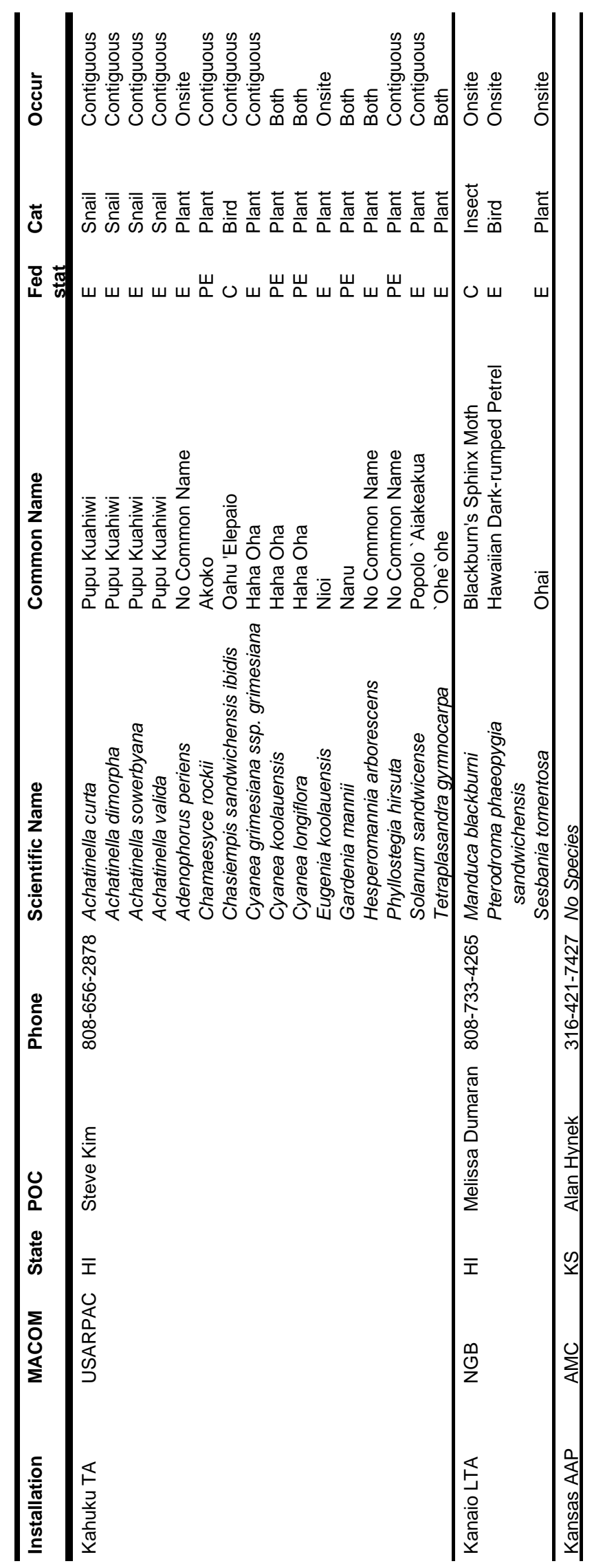




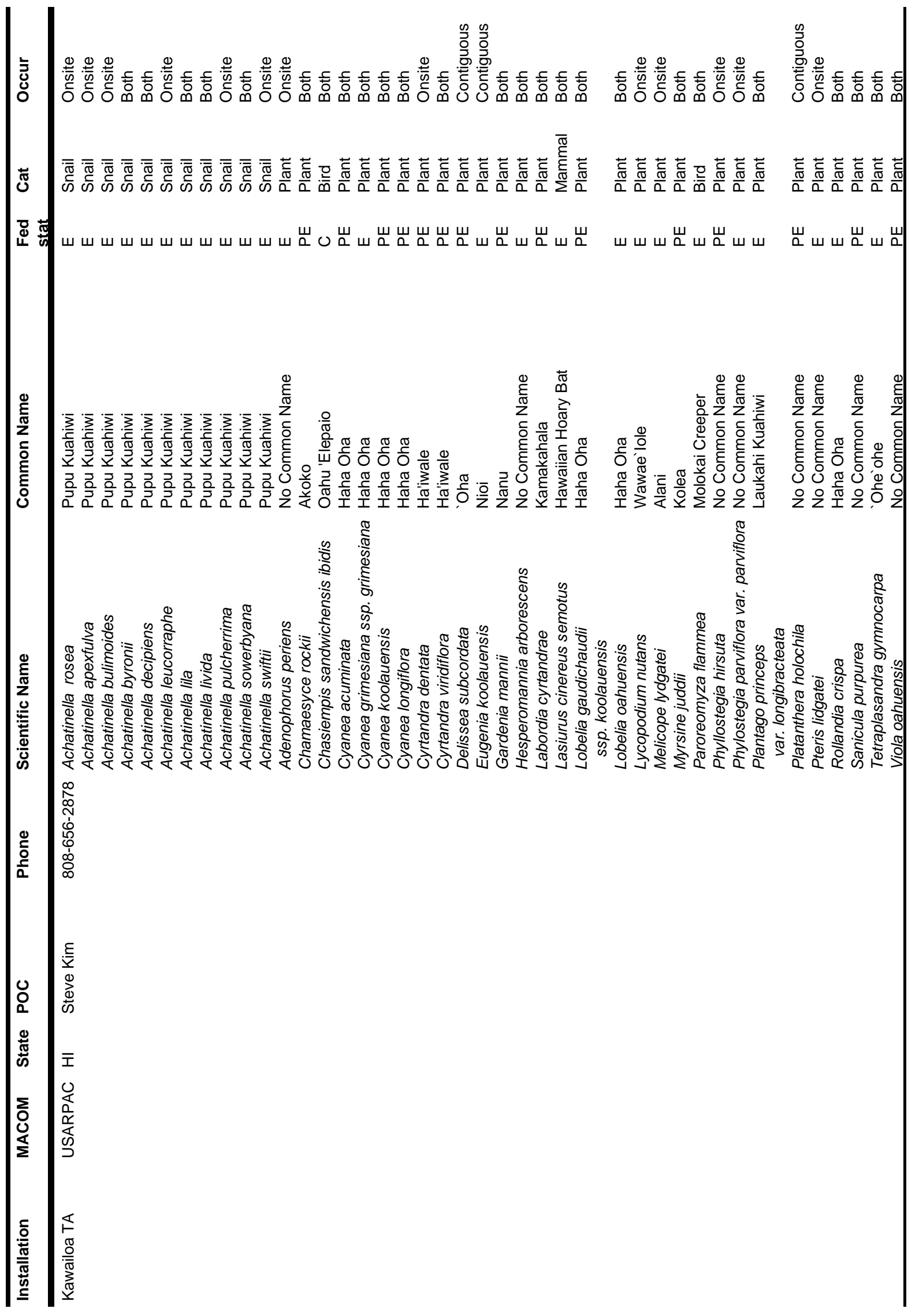




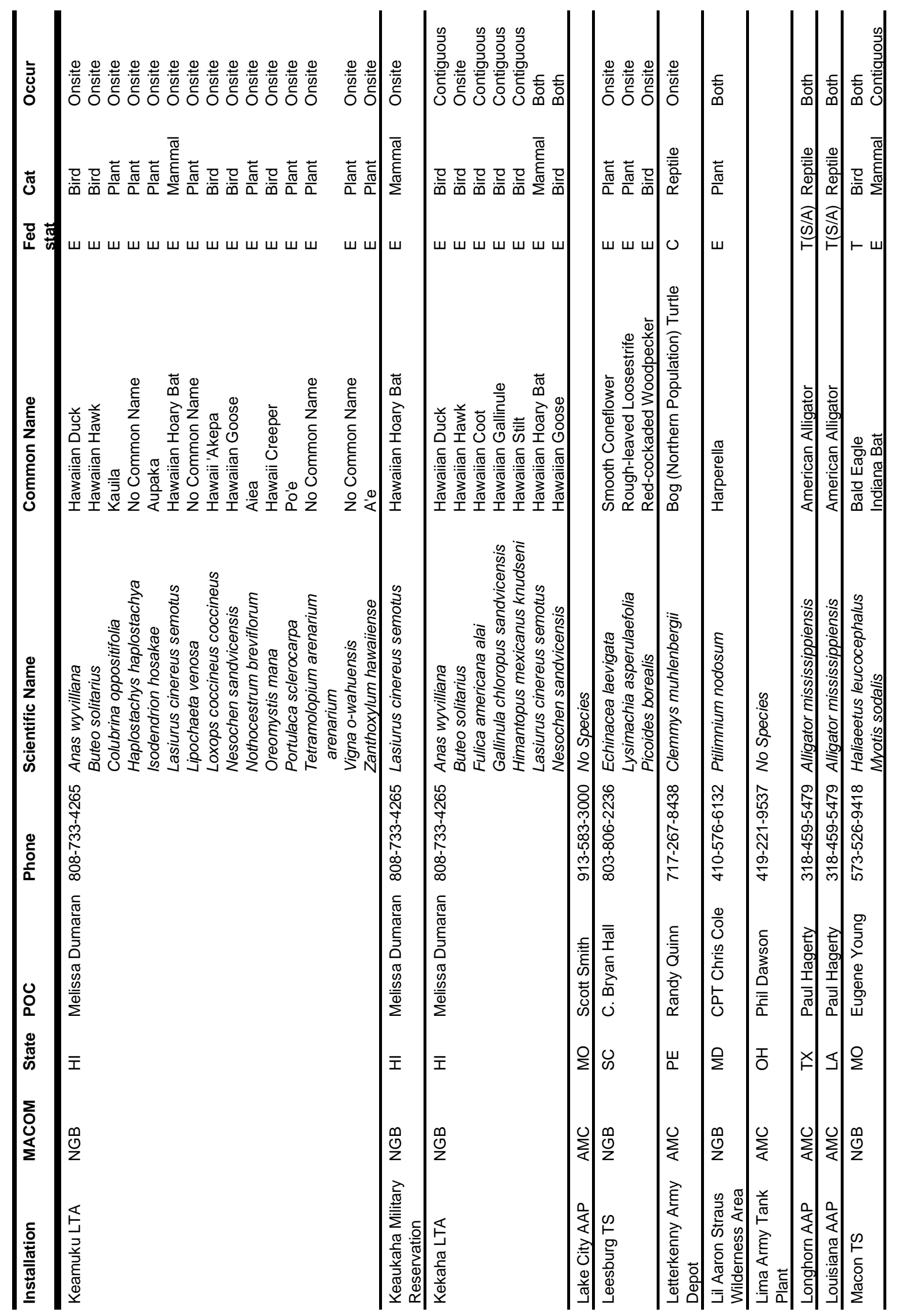




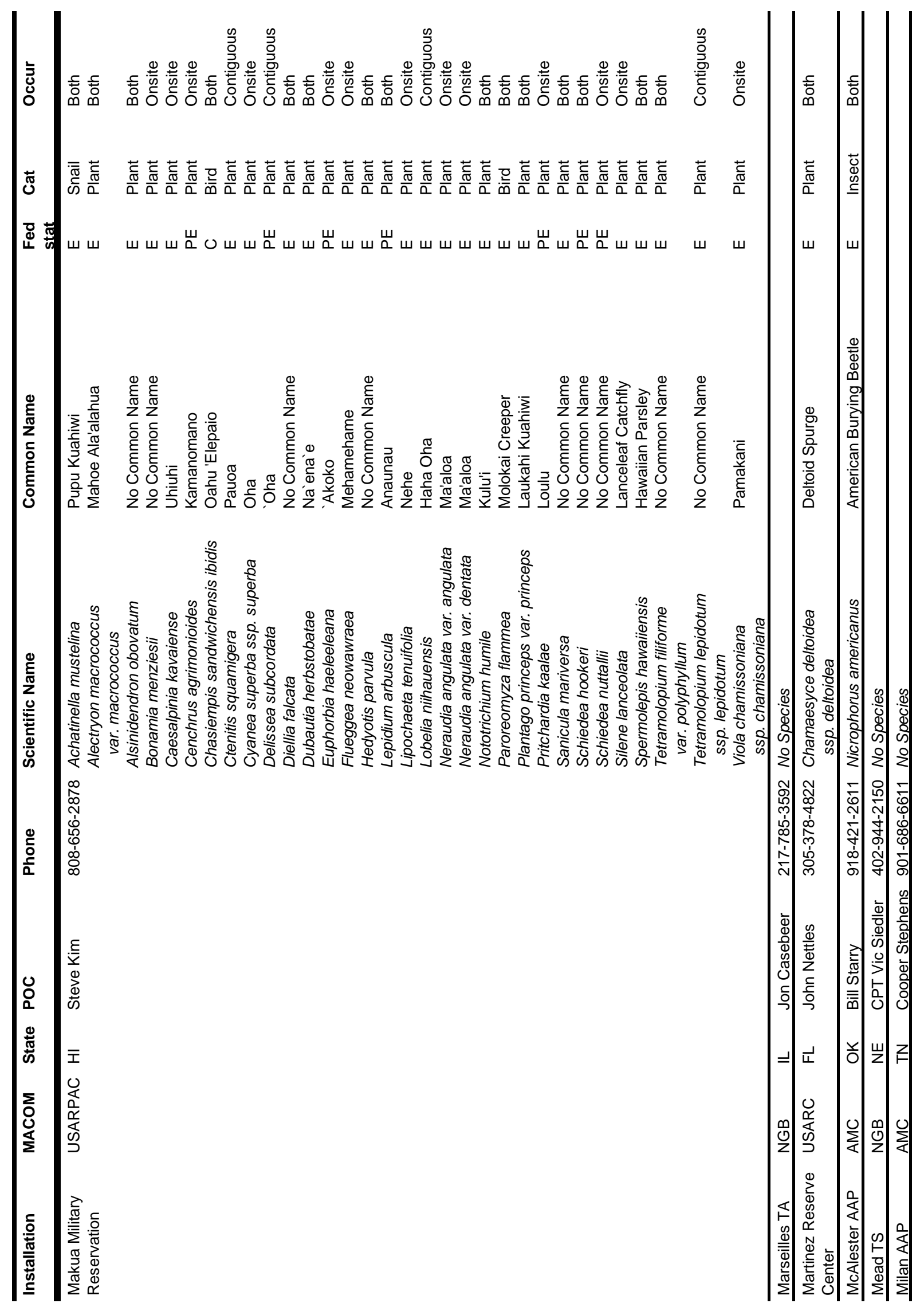




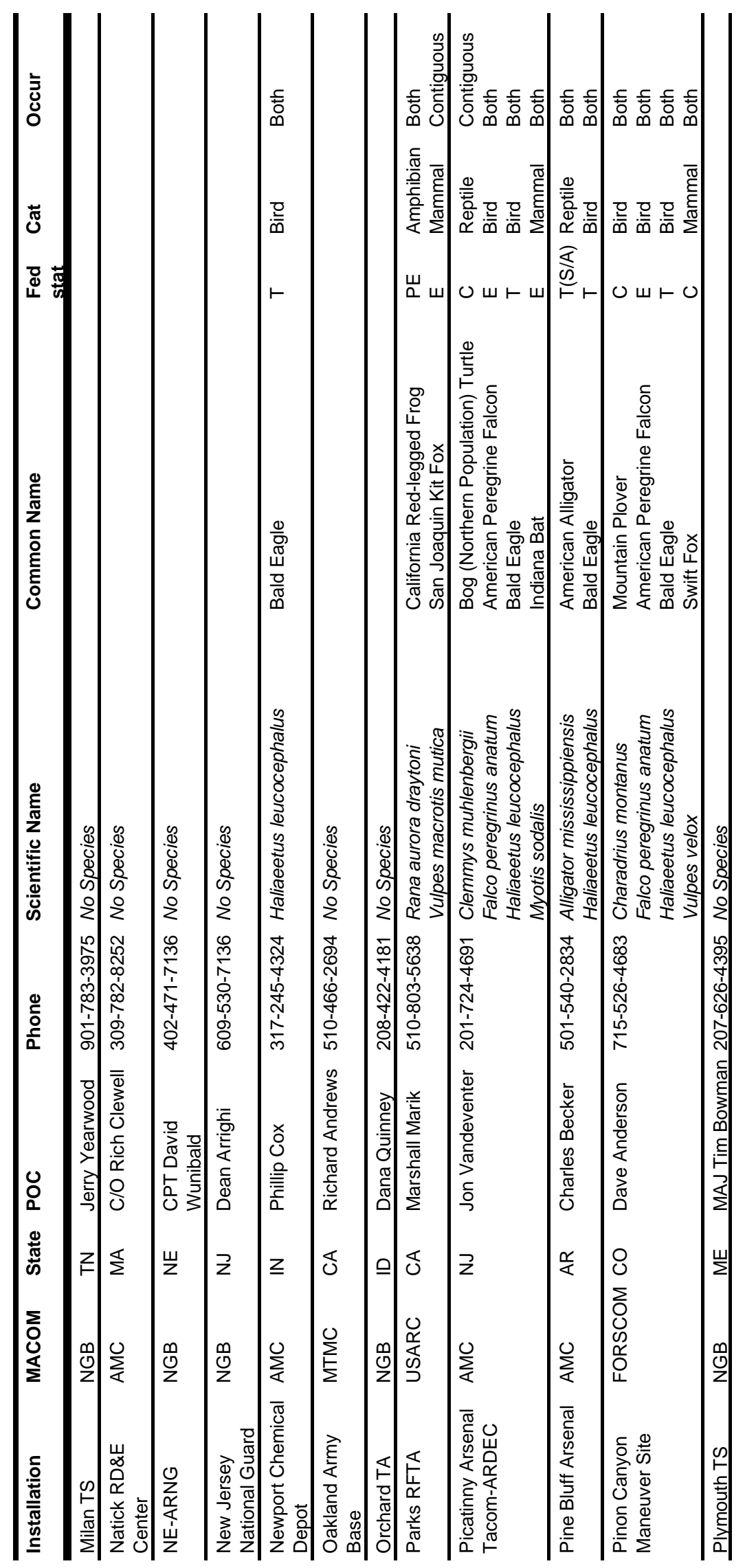




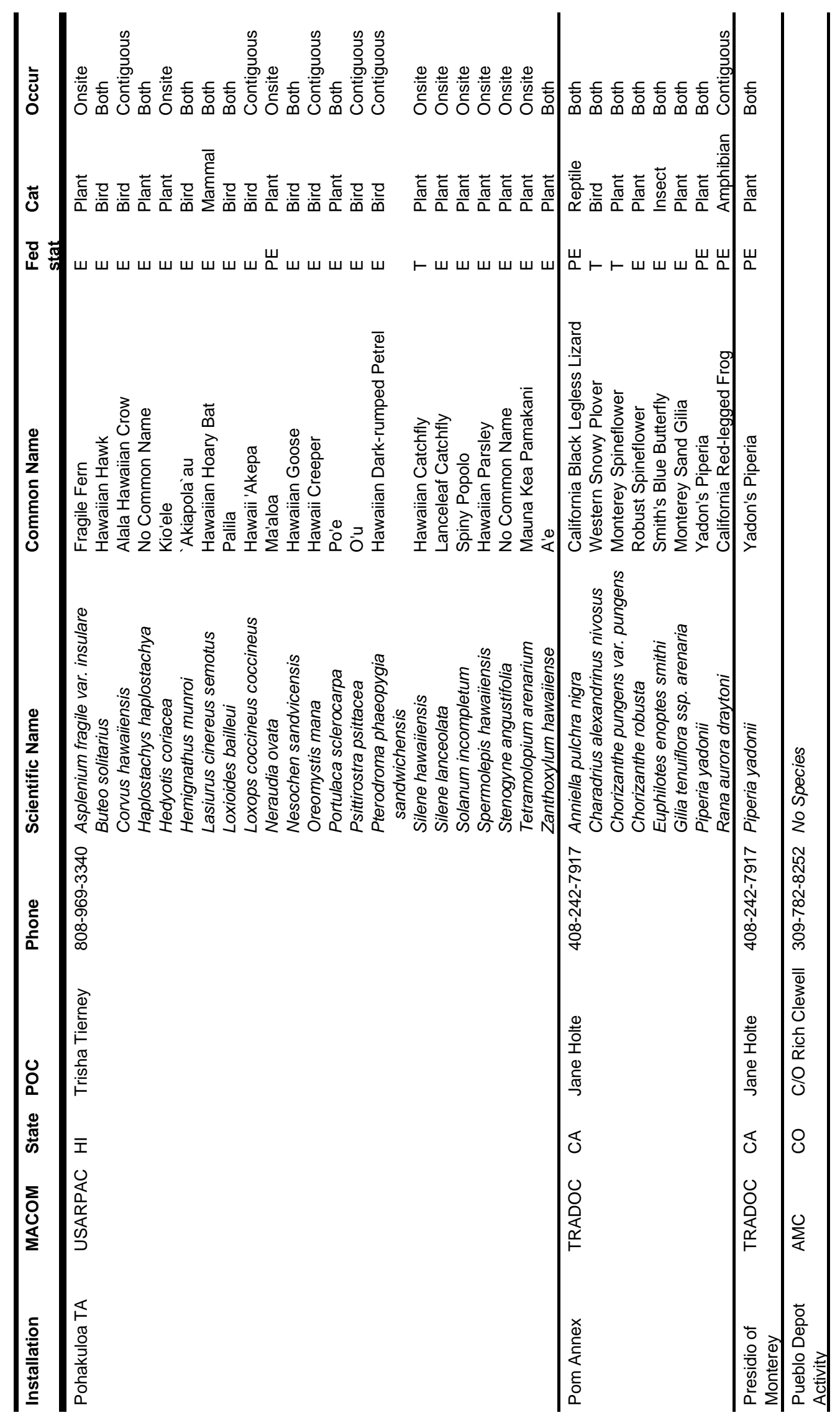




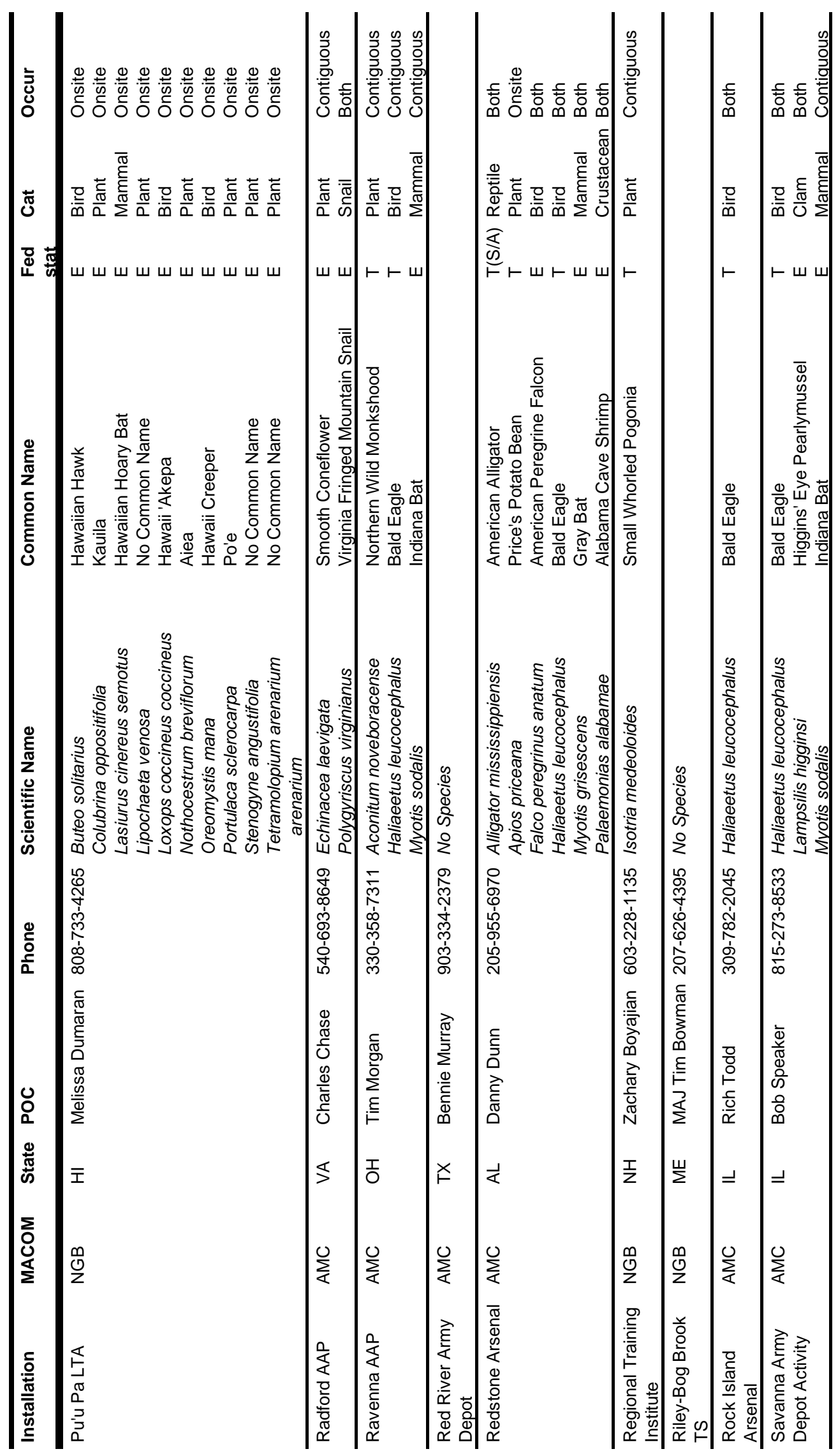




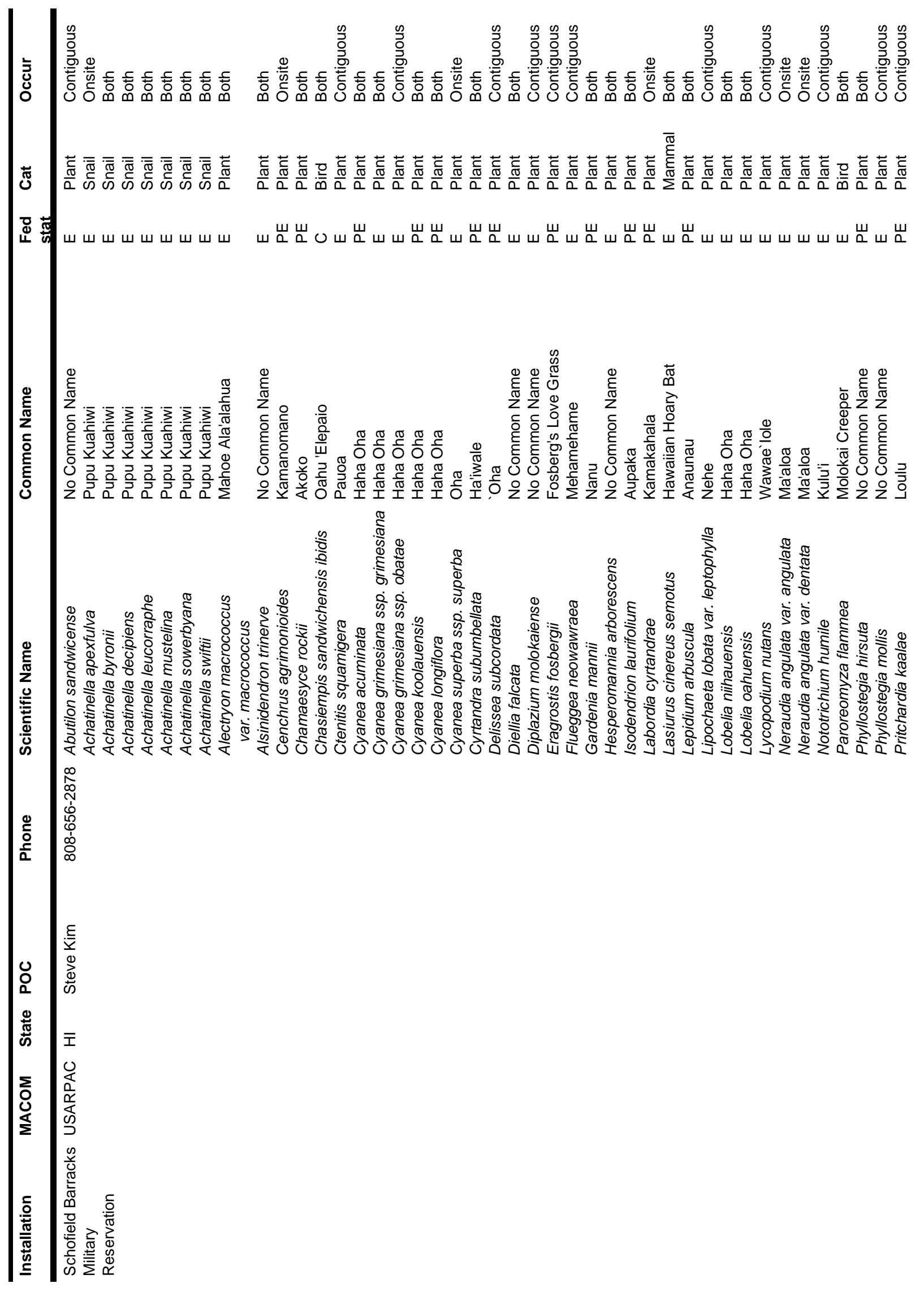




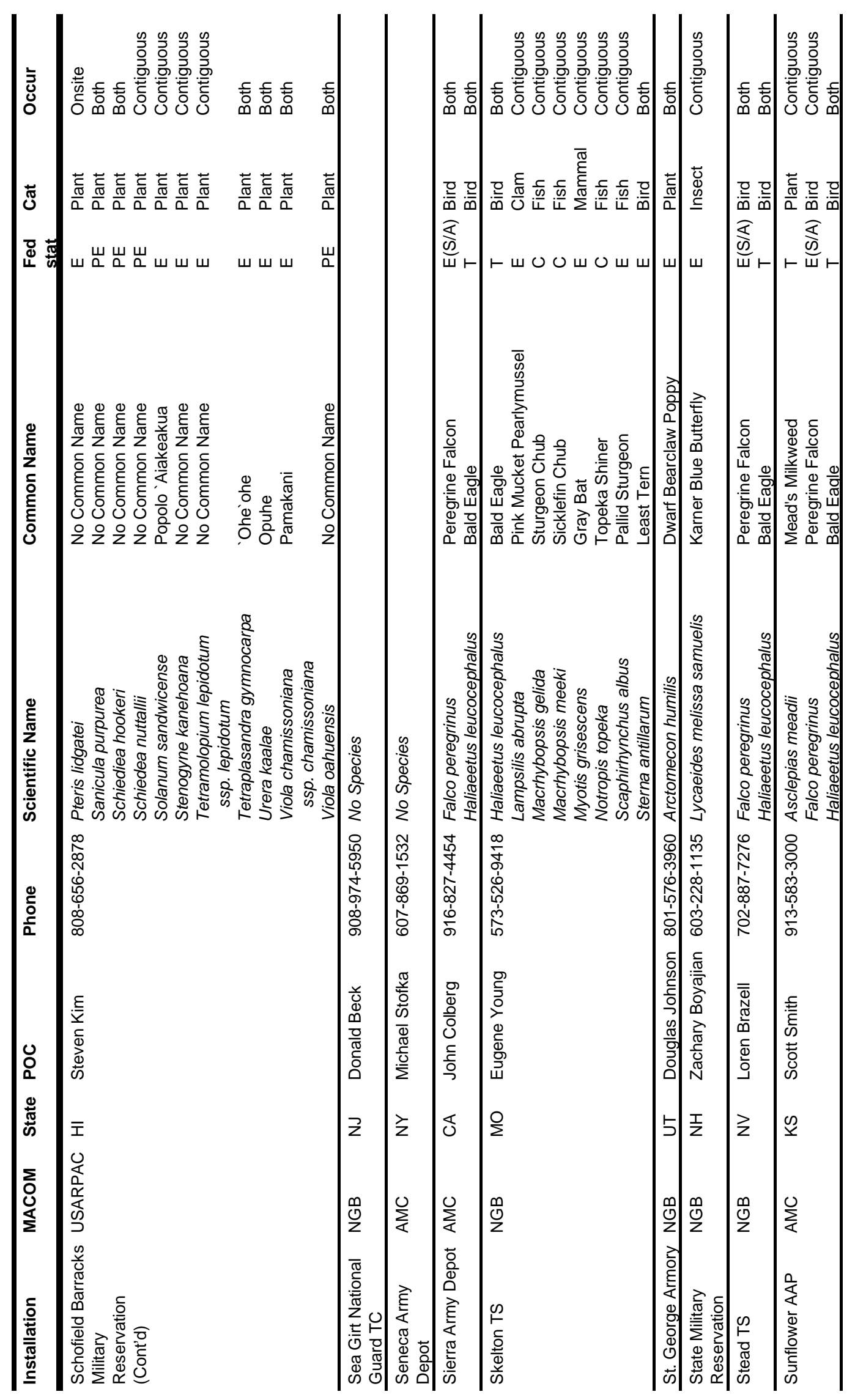




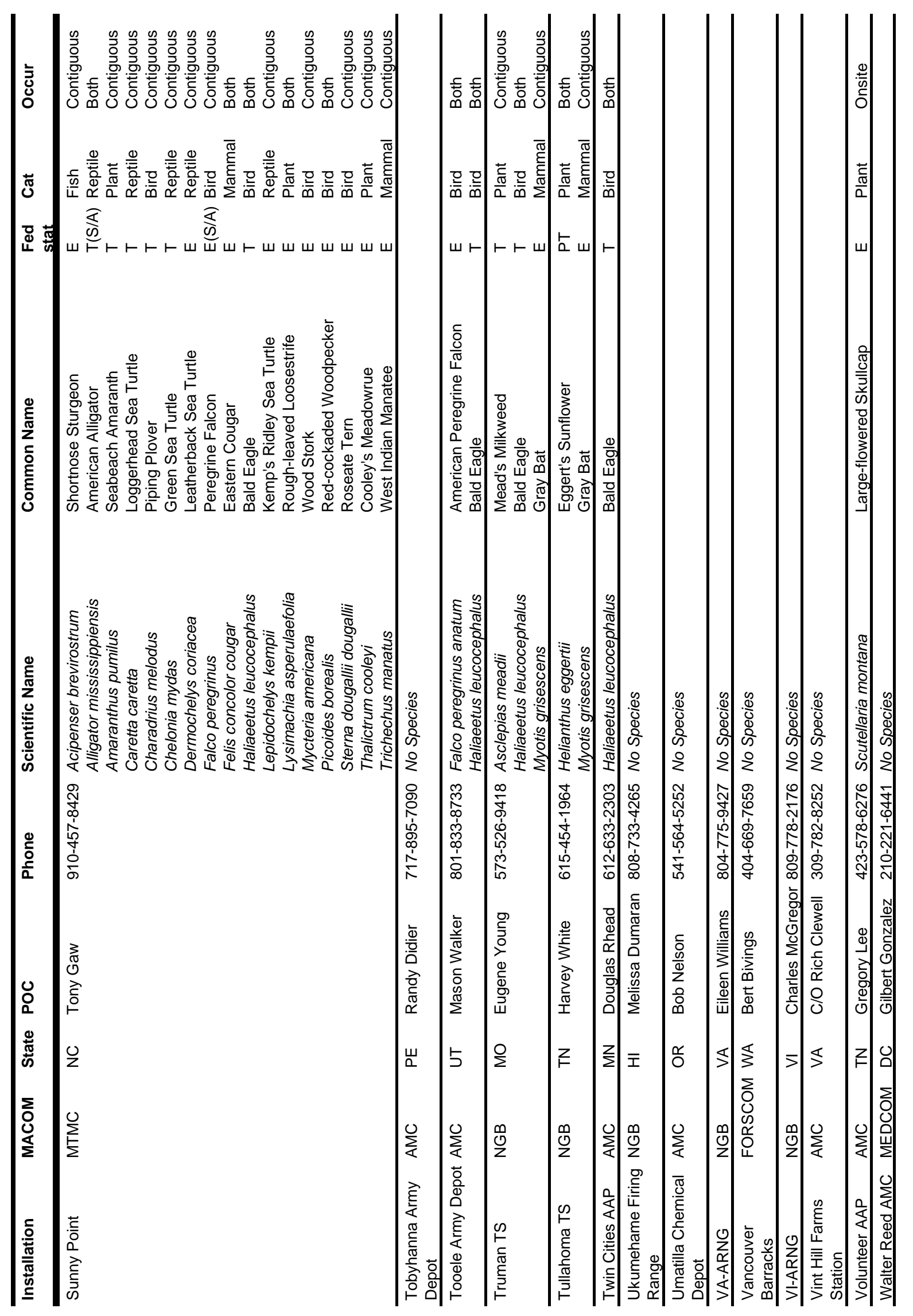




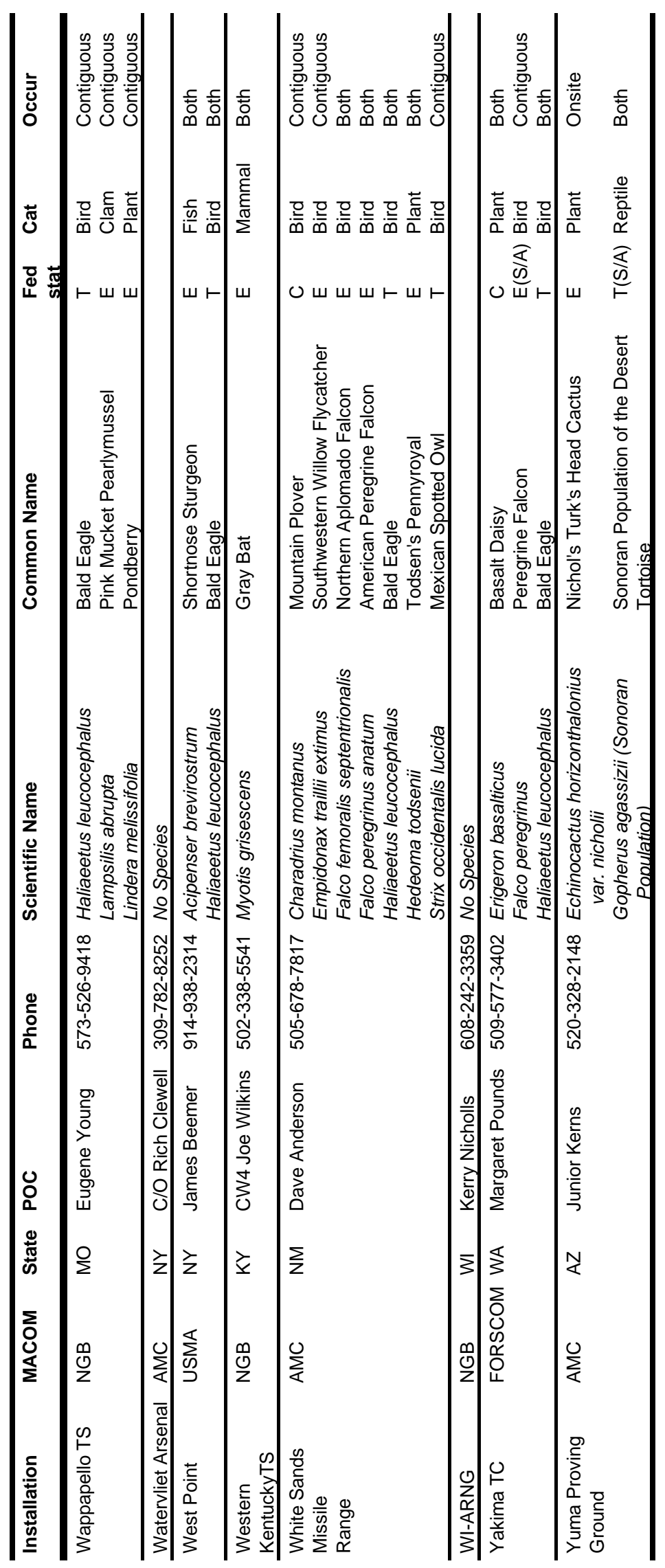




\section{Appendix B: Critical Habitat and ESMP Status for Onsite and Contiguous TEPC Species, With Corresponding Installations}

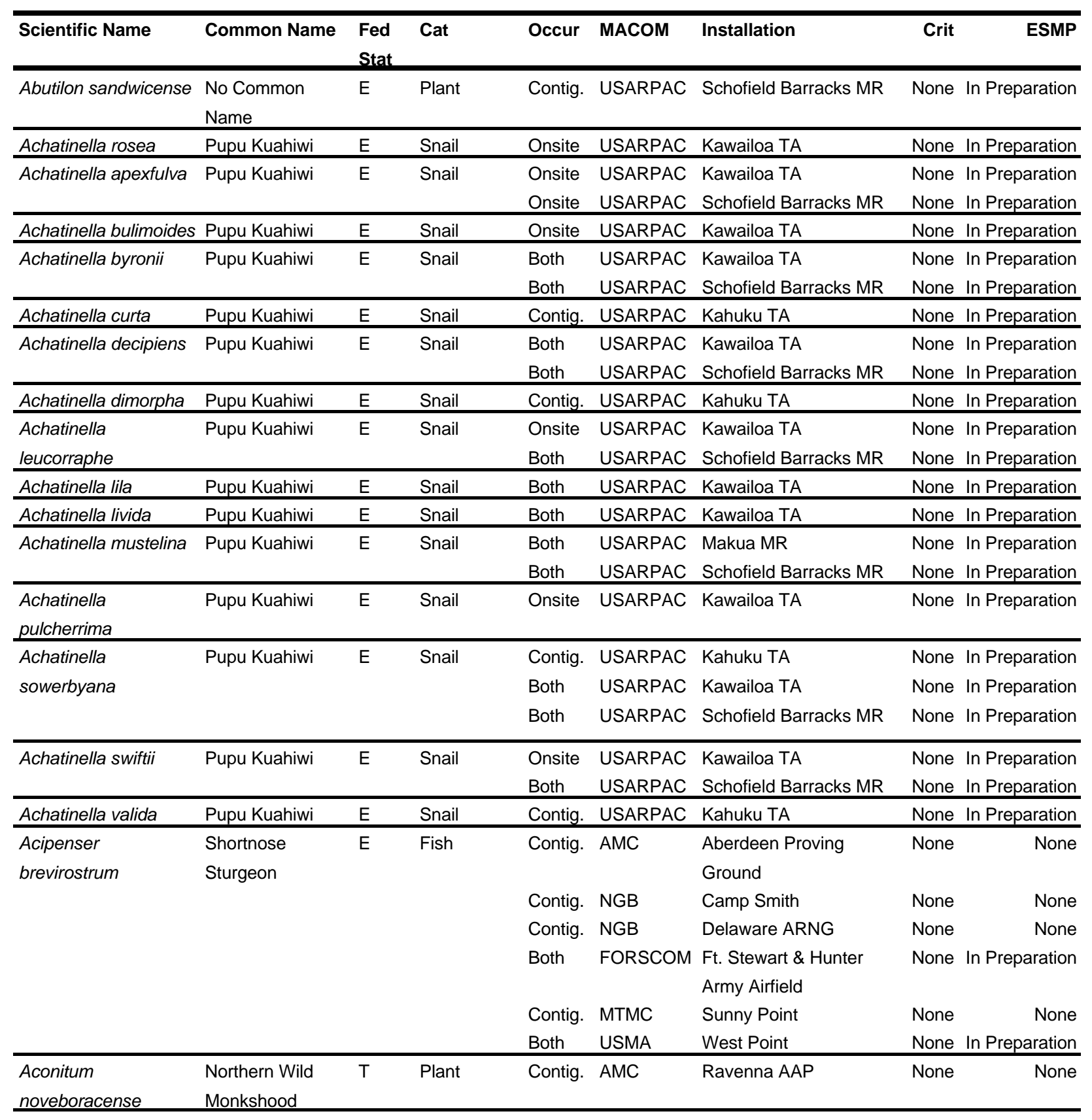




\begin{tabular}{|c|c|c|c|c|c|c|c|c|}
\hline Scientific Name & Common Name & $\begin{array}{l}\text { Fed } \\
\text { Stat }\end{array}$ & Cat & Occur & MACOM & Installation & Crit & ESMP \\
\hline Adenophorus periens & $\begin{array}{l}\text { No Common } \\
\text { Name }\end{array}$ & $\mathrm{E}$ & Plant & $\begin{array}{l}\text { Onsite } \\
\text { Onsite } \\
\end{array}$ & $\begin{array}{l}\text { USARPAC } \\
\text { USARPAC } \\
\end{array}$ & $\begin{array}{l}\text { Kahuku TA } \\
\text { Kawailoa TA }\end{array}$ & $\begin{array}{l}\text { None } \\
\text { None } \\
\end{array}$ & $\begin{array}{l}\text { In Preparation } \\
\text { In Preparation }\end{array}$ \\
\hline $\begin{array}{l}\text { Alectryon } \\
\text { macrococcus }\end{array}$ & Mahoe Ala'alahua & $E$ & Plant & $\begin{array}{l}\text { Both } \\
\text { Both } \\
\end{array}$ & $\begin{array}{l}\text { USARPAC } \\
\text { USARPAC } \\
\end{array}$ & $\begin{array}{l}\text { Makua MR } \\
\text { Schofield Barracks MR }\end{array}$ & $\begin{array}{l}\text { None } \\
\text { None } \\
\end{array}$ & $\begin{array}{l}\text { In Preparation } \\
\text { In Preparation }\end{array}$ \\
\hline $\begin{array}{l}\text { Alligator } \\
\text { mississippiensis }\end{array}$ & $\begin{array}{l}\text { American } \\
\text { Alligator }\end{array}$ & $\mathrm{T}(\mathrm{S} / \mathrm{A})$ & Reptile & $\begin{array}{l}\text { Both } \\
\text { Both }\end{array}$ & $\begin{array}{l}\text { NGB } \\
\text { TRADOC }\end{array}$ & $\begin{array}{l}\text { Camp Blanding TS } \\
\text { Ft. Benning }\end{array}$ & $\begin{array}{l}\text { None } \\
\text { None }\end{array}$ & $\begin{array}{r}\text { Completed } \\
\text { In Preparation }\end{array}$ \\
\hline & & & & Onsite & TRADOC & Ft. Jackson & None & None \\
\hline & & & & Both & TRADOC & Ft. Rucker & None & None \\
\hline & & & & Both & AMC & Longhorn AAP & None & None \\
\hline & & & & Both & AMC & Louisiana AAP & None & None \\
\hline & & & & Both & AMC & Pine Bluff Arsenal & None & None \\
\hline & & & & Both & AMC & Redstone Arsenal & None & None \\
\hline & & & & Both & MTMC & Sunny Point & None & In Preparation \\
\hline $\begin{array}{l}\text { Alsinidendron } \\
\text { obovatum }\end{array}$ & $\begin{array}{l}\text { No Common } \\
\text { Name }\end{array}$ & $\mathrm{E}$ & Plant & Both & USARPAC & Makua MR & None & In Preparation \\
\hline Alsinidendron trinerve & $\begin{array}{l}\text { No Common } \\
\text { Name }\end{array}$ & $\mathrm{E}$ & Plant & Both & USARPAC & Schofield Barracks MR & None & In Preparation \\
\hline Amaranthus pumilus & $\begin{array}{l}\text { Seabeach } \\
\text { Amaranth }\end{array}$ & $\mathrm{T}$ & Plant & Contig. & MTMC & Sunny Point & None & None \\
\hline Amblyopsis rosae & Ozark Cavefish & $\mathrm{T}$ & Fish & Contig. & NGB & Camp Crowder & None & In Preparation \\
\hline $\begin{array}{l}\text { Ambystoma } \\
\text { californiense }\end{array}$ & $\begin{array}{l}\text { Ambystoma } \\
\text { Californiense }\end{array}$ & C & Amphibian & $\begin{array}{l}\text { Both } \\
\text { Both }\end{array}$ & $\begin{array}{l}\text { TRADOC } \\
\text { USARC }\end{array}$ & $\begin{array}{l}\text { Ft. Huachuca } \\
\text { Ft. Hunter Liggett }\end{array}$ & $\begin{array}{l}\mathrm{NA} \\
\mathrm{NA}\end{array}$ & $\begin{array}{l}\text { NA } \\
\text { NA }\end{array}$ \\
\hline Anas wyvilliana & Hawaiian Duck & $\mathrm{E}$ & Bird & Onsite & NGB & $\begin{array}{l}\text { Aahoaka \& Wailua } \\
\text { River LTA }\end{array}$ & None & None \\
\hline & & & & Both & NGB & Anahola LTA & None & None \\
\hline & & & & Both & USARPAC & Dillingham TA & None & In Preparation \\
\hline & & & & Onsite & NGB & Keamuku LTA & None & None \\
\hline & & & & Contig. & NGB & Kekaha LTA & None & None \\
\hline Anniella pulchra nigra & $\begin{array}{l}\text { California Black } \\
\text { Legless Lizard } \\
\end{array}$ & PE & Reptile & Both & TRADOC & Pom Annex & NA & Completed \\
\hline $\begin{array}{l}\text { Aphelocoma } \\
\text { coerulescens }\end{array}$ & Florida Scrub Jay & $\mathrm{T}$ & Bird & Both & NGB & Camp Blanding TS & None & Completed \\
\hline Apios priceana & $\begin{array}{l}\text { Price's Potato } \\
\text { Bean }\end{array}$ & $\mathrm{T}$ & Plant & $\begin{array}{l}\text { Contig. } \\
\text { Onsite }\end{array}$ & $\begin{array}{l}\text { FORSCOM } \\
\text { AMC } \\
\end{array}$ & $\begin{array}{l}\text { Ft. Campbell } \\
\text { Redstone Arsenal }\end{array}$ & $\begin{array}{l}\text { None } \\
\text { None } \\
\end{array}$ & $\begin{array}{r}\text { None } \\
\text { In Preparation }\end{array}$ \\
\hline Arctomecon humilis & $\begin{array}{l}\text { Dwarf Bearclaw } \\
\text { Poppy }\end{array}$ & $E$ & Plant & Both & NGB & St. George Armory & None & Completed \\
\hline Asclepias meadii & Mead's Milkweed & $\mathrm{T}$ & Plant & $\begin{array}{l}\text { Contig. } \\
\text { Contig. } \\
\text { Contig. }\end{array}$ & $\begin{array}{l}\text { NGB } \\
\text { AMC } \\
\text { NGB } \\
\end{array}$ & $\begin{array}{l}\text { Camp Clark } \\
\text { Sunflower AAP } \\
\text { Truman TS } \\
\end{array}$ & $\begin{array}{l}\text { None } \\
\text { None } \\
\text { None } \\
\end{array}$ & $\begin{array}{l}\text { None } \\
\text { None } \\
\text { None } \\
\end{array}$ \\
\hline $\begin{array}{l}\text { Asplenium fragile } \\
\text { var. insulare }\end{array}$ & Fragile Fern & $E$ & Plant & Onsite & USARPAC & Pohakuloa TA & None & In Preparation \\
\hline $\begin{array}{l}\text { Astragalus } \\
\text { jaegerianus } \\
\end{array}$ & $\begin{array}{l}\text { Lane Mountain } \\
\text { Milk Vetch } \\
\end{array}$ & C & Plant & Both & FORSCOM & Ft. Irwin & NA & NA \\
\hline Banara vanderbiltii & Palo de Ramon & $\mathrm{E}$ & Plant & Onsite & FORSCOM & Ft. Buchanan & None & None \\
\hline Bonamia menziesii & $\begin{array}{l}\text { No Common } \\
\text { Name }\end{array}$ & $E$ & Plant & Onsite & USARPAC & Makua MR & None & In Preparation \\
\hline Branchinecta lynchi & $\begin{array}{l}\text { Vernal Pool Fairy } \\
\text { Shrimp }\end{array}$ & $\mathrm{T}$ & Crustacean & $\begin{array}{l}\text { Both } \\
\text { Onsite }\end{array}$ & $\begin{array}{l}\text { NGB } \\
\text { USARC }\end{array}$ & $\begin{array}{l}\text { Camp Roberts } \\
\text { Ft. Hunter Liggett }\end{array}$ & $\begin{array}{l}\text { None } \\
\text { None }\end{array}$ & $\begin{array}{r}\text { None } \\
\text { In Preparation }\end{array}$ \\
\hline
\end{tabular}




\begin{tabular}{|c|c|c|c|c|c|c|c|c|}
\hline Scientific Name & Common Name & $\begin{array}{l}\text { Fed } \\
\text { Stat }\end{array}$ & Cat & Occur & MACOM & Installation & Crit & ESMP \\
\hline $\begin{array}{l}\text { Bufo microscaphus } \\
\text { californicus }\end{array}$ & $\begin{array}{l}\text { Arroyo } \\
\text { Southwestern } \\
\text { Toad }\end{array}$ & $E$ & Amphibian & Onsite & USARC & Ft. Hunter Liggett & None & In Preparation \\
\hline Buteo solitarius & Hawaiian Hawk & $E$ & Bird & $\begin{array}{l}\text { Onsite } \\
\text { Onsite } \\
\text { Both } \\
\text { Onsite }\end{array}$ & $\begin{array}{l}\text { NGB } \\
\text { NGB } \\
\text { USARPAC } \\
\text { NGB }\end{array}$ & $\begin{array}{l}\text { Keamuku LTA } \\
\text { Kekaha LTA } \\
\text { Pohakuloa TA } \\
\text { Pu'u Pa LTA }\end{array}$ & $\begin{array}{l}\text { None } \\
\text { None } \\
\text { None } \\
\text { None }\end{array}$ & $\begin{array}{r}\text { None } \\
\text { None } \\
\text { In Preparation } \\
\text { None } \\
\end{array}$ \\
\hline Buxus vahlii & Vahl Boxwood & $\mathrm{E}$ & Plant & Onsite & FORSCOM & Ft. Buchanan & None & None \\
\hline Caesalpinia kavaiense & Uhiuhi & $E$ & Plant & Onsite & USARPAC & Makua MR & None & In Preparation \\
\hline Canis lupus & Gray Wolf & $\mathrm{T}$ & Mammal & $\begin{array}{l}\text { Both } \\
\text { Contig. }\end{array}$ & $\begin{array}{l}\text { NGB } \\
\text { USARC }\end{array}$ & $\begin{array}{l}\text { Camp Ripley } \\
\text { Ft. McCoy }\end{array}$ & $\begin{array}{l}\text { None } \\
\text { None }\end{array}$ & $\begin{array}{r}\text { In Preparation } \\
\text { None } \\
\end{array}$ \\
\hline Caretta caretta & $\begin{array}{l}\text { Loggerhead Sea } \\
\text { Turtle }\end{array}$ & $\mathrm{T}$ & Reptile & Contig. & MTMC & Sunny Point & None & None \\
\hline $\begin{array}{l}\text { Cenchrus } \\
\text { agrimonioides }\end{array}$ & Kamanomano & PE & Plant & $\begin{array}{l}\text { Onsite } \\
\text { Onsite } \\
\end{array}$ & $\begin{array}{l}\text { USARPAC } \\
\text { USARPAC } \\
\end{array}$ & $\begin{array}{l}\text { Makua MR } \\
\text { Schofield Barracks MR }\end{array}$ & $\begin{array}{l}\text { NA } \\
\text { NA } \\
\end{array}$ & $\begin{array}{l}\text { In Preparation } \\
\text { In Preparation } \\
\end{array}$ \\
\hline $\begin{array}{l}\text { Chamaesyce } \\
\text { deltoidea ssp. } \\
\text { deltoidea }\end{array}$ & Deltoid Spurge & $\mathrm{E}$ & Plant & Both & USARC & $\begin{array}{l}\text { Martinez Reserve Cen- } \\
\text { ter }\end{array}$ & None & Completed \\
\hline Chamaesyce rockii & Akoko & $\mathrm{PE}$ & Plant & $\begin{array}{l}\text { Contig. } \\
\text { Both } \\
\text { Both }\end{array}$ & $\begin{array}{l}\text { USARPAC } \\
\text { USARPAC } \\
\text { USARPAC } \\
\end{array}$ & $\begin{array}{l}\text { Kahuku TA } \\
\text { Kawailoa TA } \\
\text { Schofield Barracks MR }\end{array}$ & $\begin{array}{l}\text { NA } \\
\text { NA } \\
\text { NA }\end{array}$ & $\begin{array}{l}\text { In Preparation } \\
\text { In Preparation } \\
\text { In Preparation } \\
\end{array}$ \\
\hline $\begin{array}{l}\text { Charadrius } \\
\text { alexandrinus nivosus }\end{array}$ & $\begin{array}{l}\text { Western Snowy } \\
\text { Plover }\end{array}$ & $\mathrm{T}$ & Bird & Both & TRADOC & Pom Annex & None & Completed \\
\hline Charadrius montanus & Mountain Plover & $\mathrm{C}$ & Bird & $\begin{array}{l}\text { Contig. } \\
\text { Both } \\
\text { Both } \\
\text { Contig. }\end{array}$ & $\begin{array}{l}\text { AMC } \\
\text { FORSCOM } \\
\text { FORSCOM } \\
\text { AMC }\end{array}$ & $\begin{array}{l}\text { Dugway Proving } \\
\text { Ground } \\
\text { Ft. Carson } \\
\text { Pinon Canyon MS } \\
\text { White Sands Missile } \\
\text { Rng. }\end{array}$ & $\begin{array}{l}\text { NA } \\
\text { NA } \\
\text { NA }\end{array}$ & $\begin{array}{l}\text { NA } \\
\text { NA } \\
\text { NA }\end{array}$ \\
\hline $\begin{array}{l}\text { Chasiempis } \\
\text { sandwichensis ibidis }\end{array}$ & Oahu 'Elepaio & C & Bird & $\begin{array}{l}\text { Contig. } \\
\text { Both } \\
\text { Both } \\
\text { Both }\end{array}$ & $\begin{array}{l}\text { USARPAC } \\
\text { USARPAC } \\
\text { USARPAC } \\
\text { USARPAC } \\
\end{array}$ & $\begin{array}{l}\text { Kahuku TA } \\
\text { Kawailoa TA } \\
\text { Makua MR } \\
\text { Schofield Barracks MR }\end{array}$ & $\begin{array}{l}\text { NA } \\
\text { NA } \\
\text { NA } \\
\text { NA }\end{array}$ & $\begin{array}{l}\text { NA } \\
\text { NA } \\
\text { NA } \\
\text { NA }\end{array}$ \\
\hline Chelonia mydas & Green Sea Turtle & $\mathrm{T}$ & Reptile & Contig. & MTMC & Sunny Point & None & None \\
\hline $\begin{array}{l}\text { Chlorogalium } \\
\text { purpureum } \\
\text { var. purpureum }\end{array}$ & Purple Amole & C & Plant & Onsite & USARC & Ft. Hunter Liggett & NA & NA \\
\hline $\begin{array}{l}\text { Chorizanthe pungens } \\
\text { var. pungens }\end{array}$ & $\begin{array}{l}\text { Monterey } \\
\text { Spineflower }\end{array}$ & $\mathrm{T}$ & Plant & Both & TRADOC & Pom Annex & None & Completed \\
\hline Chorizanthe robusta & $\begin{array}{l}\text { Robust } \\
\text { Spineflower }\end{array}$ & $E$ & Plant & Both & TRADOC & Pom Annex & None & Completed \\
\hline Cicindela puritana & $\begin{array}{l}\text { Puritan Tiger Bee- } \\
\text { tle }\end{array}$ & $\mathrm{T}$ & Insect & Contig. & AMC & $\begin{array}{l}\text { Aberdeen Proving } \\
\text { Ground }\end{array}$ & None & None \\
\hline $\begin{array}{l}\text { Cirsium fontinale } \\
\text { var. obispoense }\end{array}$ & $\begin{array}{l}\text { Chorro Creek Bog } \\
\text { Thistle }\end{array}$ & $E$ & Plant & Both & NGB & Camp San Luis Obispo & None & In Preparation \\
\hline
\end{tabular}




\begin{tabular}{|c|c|c|c|c|c|c|c|c|}
\hline Scientific Name & Common Name & $\begin{array}{l}\text { Fed } \\
\text { Stat }\end{array}$ & Cat & Occur & MACOM & Installation & Crit & ESMP \\
\hline \multirow[t]{2}{*}{$\begin{array}{l}\text { Clemmys } \\
\text { muhlenbergii }\end{array}$} & $\begin{array}{l}\text { Bog (Northern } \\
\text { Population) Turtle }\end{array}$ & $\mathrm{C}$ & Reptile & Onsite & AMC & $\begin{array}{l}\text { Letterkenny Army } \\
\text { Depot }\end{array}$ & NA & NA \\
\hline & & & & Contig. & AMC & $\begin{array}{l}\text { Picatinny Arsenal } \\
\text { Tacom-ARDEC }\end{array}$ & NA & NA \\
\hline Colubrina oppositifolia & Kauila & $\mathrm{E}$ & Plant & $\begin{array}{l}\text { Onsite } \\
\text { Onsite }\end{array}$ & $\begin{array}{l}\text { NGB } \\
\text { NGB }\end{array}$ & $\begin{array}{l}\text { Keamuku LTA } \\
\text { Pu'u Pa LTA }\end{array}$ & $\begin{array}{l}\text { None } \\
\text { None }\end{array}$ & $\begin{array}{l}\text { None } \\
\text { None }\end{array}$ \\
\hline Corvus hawaiiensis & $\begin{array}{l}\text { Alala Hawaiian } \\
\text { Crow }\end{array}$ & $E$ & Bird & Contig. & USARPAC & Pohakuloa TA & None & In Preparation \\
\hline $\begin{array}{l}\text { Coryphantha sneedii } \\
\text { var. sneedii }\end{array}$ & $\begin{array}{l}\text { Sneed Pincushion } \\
\text { Cactus }\end{array}$ & $E$ & Plant & Both & TRADOC & Ft. Bliss & None & In Preparation \\
\hline Ctenitis squamigera & Pauoa & $E$ & Plant & $\begin{array}{l}\text { Contig. } \\
\text { Contig. }\end{array}$ & $\begin{array}{l}\text { USARPAC } \\
\text { USARPAC } \\
\end{array}$ & $\begin{array}{l}\text { Makua MR } \\
\text { Schofield Barracks MR }\end{array}$ & $\begin{array}{l}\text { None } \\
\text { None }\end{array}$ & $\begin{array}{l}\text { In Preparation } \\
\text { In Preparation }\end{array}$ \\
\hline Cyanea acuminata & Haha Oha & $\mathrm{PE}$ & Plant & $\begin{array}{l}\text { Both } \\
\text { Both }\end{array}$ & $\begin{array}{l}\text { USARPAC } \\
\text { USARPAC } \\
\end{array}$ & $\begin{array}{l}\text { Kawailoa TA } \\
\text { Schofield Barracks MR }\end{array}$ & $\begin{array}{r}\text { None } \\
\text { NA } \\
\end{array}$ & $\begin{array}{l}\text { In Preparation } \\
\text { In Preparation }\end{array}$ \\
\hline ssp. grimesiana & Haha Oha & $E$ & Plant & $\begin{array}{l}\text { Contig. } \\
\text { Both } \\
\text { Both } \\
\text { Contig. }\end{array}$ & $\begin{array}{l}\text { USARPAC } \\
\text { USARPAC } \\
\text { USARPAC } \\
\text { USARPAC } \\
\end{array}$ & $\begin{array}{l}\text { Kahuku TA } \\
\text { Kawailoa TA } \\
\text { Schofield Barracks MR } \\
\text { Schofield Barracks MR }\end{array}$ & $\begin{array}{l}\text { None } \\
\text { None } \\
\text { None } \\
\text { None }\end{array}$ & $\begin{array}{l}\text { In Preparation } \\
\text { In Preparation } \\
\text { In Preparation } \\
\text { In Preparation }\end{array}$ \\
\hline Cyanea koolauensis & Haha Oha & PE & Plant & $\begin{array}{l}\text { Both } \\
\text { Both } \\
\text { Both }\end{array}$ & $\begin{array}{l}\text { USARPAC } \\
\text { USARPAC } \\
\text { USARPAC } \\
\end{array}$ & $\begin{array}{l}\text { Kahuku TA } \\
\text { Kawailoa TA } \\
\text { Schofield Barracks MR }\end{array}$ & $\begin{array}{l}\text { NA } \\
\text { NA } \\
\text { NA } \\
\end{array}$ & $\begin{array}{l}\text { In Preparation } \\
\text { In Preparation } \\
\text { In Preparation }\end{array}$ \\
\hline Cyanea longiflora & Haha Oha & PE & Plant & $\begin{array}{l}\text { Both } \\
\text { Both } \\
\text { Both }\end{array}$ & $\begin{array}{l}\text { USARPAC } \\
\text { USARPAC } \\
\text { USARPAC } \\
\end{array}$ & $\begin{array}{l}\text { Kahuku TA } \\
\text { Kawailoa TA } \\
\text { Schofield Barracks MR }\end{array}$ & $\begin{array}{l}\text { NA } \\
\text { NA } \\
\text { NA } \\
\end{array}$ & $\begin{array}{l}\text { In Preparation } \\
\text { In Preparation } \\
\text { In Preparation }\end{array}$ \\
\hline $\begin{array}{l}\text { Cyanea superba } \\
\text { spp. superba }\end{array}$ & Oha & $\mathrm{E}$ & Plant & $\begin{array}{l}\text { Onsite } \\
\text { Onsite } \\
\end{array}$ & $\begin{array}{l}\text { USARPAC } \\
\text { USARPAC } \\
\end{array}$ & $\begin{array}{l}\text { Makua MR } \\
\text { Schofield Barracks MR }\end{array}$ & $\begin{array}{l}\text { None } \\
\text { None }\end{array}$ & $\begin{array}{l}\text { In Preparation } \\
\text { In Preparation }\end{array}$ \\
\hline Cynomys parvidens & Utah Prairie Dog & $\mathrm{T}$ & Mammal & Contig. & NGB & Cedar City Armory & None & None \\
\hline \multirow[t]{2}{*}{$\begin{array}{l}\text { Cyperus } \\
\text { trachysanthos }\end{array}$} & Pu`uka`a & $\mathrm{PE}$ & Plant & Onsite & USARPAC & Dillingham TA & NA & In Preparation \\
\hline & & & & Onsite & NGB & Ft. Ruger & NA & None \\
\hline Cyprinella caerulea & Blue Shiner & $\mathrm{T}$ & Fish & Both & TRADOC & Ft. McClellan & None & Completed \\
\hline Cyrtandra dentata & Ha'iwale & $\mathrm{PE}$ & Plant & Onsite & USARPAC & Kawailoa TA & NA & In Preparation \\
\hline $\begin{array}{l}\text { Cyrtandra } \\
\text { subumbellata }\end{array}$ & Ha'iwale & $\mathrm{PE}$ & Plant & Both & USARPAC & Schofield Barracks MR & NA & In Preparation \\
\hline Cyrtandra viridiflora & Ha'iwale & $\mathrm{PE}$ & Plant & Both & USARPAC & Kawailoa TA & NA & In Preparation \\
\hline Daphnopsis hellerana & $\begin{array}{l}\text { No Common } \\
\text { Name }\end{array}$ & $\mathrm{E}$ & Plant & Onsite & FORSCOM & Ft. Buchanan & None & None \\
\hline \multirow[t]{4}{*}{ Delissea subcordata } & 'Oha & $\mathrm{PE}$ & Bird & Onsite & AMC & $\begin{array}{l}\text { Jefferson Proving } \\
\text { Ground }\end{array}$ & NA & None \\
\hline & & & & Contig. & USARPAC & Kawailoa TA & NA & In Preparation \\
\hline & & & & Contig. & USARPAC & Makua MR & NA & In Preparation \\
\hline & & & & Contig. & USARPAC & Schofield Barracks MR & NA & In Preparation \\
\hline Dendroica chrysoparia & $\begin{array}{l}\text { Golden-cheeked } \\
\text { Warbler }\end{array}$ & $E$ & Bird & $\begin{array}{l}\text { Both } \\
\text { Both }\end{array}$ & $\begin{array}{l}\text { FORSCOM } \\
\text { MEDCOM }\end{array}$ & $\begin{array}{l}\text { Ft. Hood } \\
\text { Ft. Sam Houston }\end{array}$ & $\begin{array}{l}\text { None } \\
\text { None }\end{array}$ & $\begin{array}{l}\text { In Preparation } \\
\text { In Preparation }\end{array}$ \\
\hline Dendroica kirtlandii & Kirtland's Warbler & $\mathrm{E}$ & Bird & Both & NGB & Camp Grayling & None & Completed \\
\hline Dermochelys coriacea & $\begin{array}{l}\text { Leatherback Sea } \\
\text { Turtle }\end{array}$ & $\mathrm{E}$ & Reptile & Contig. & MTMC & Sunny Point & None & None \\
\hline \multirow[t]{2}{*}{ Diellia falcata } & No Common & $\mathrm{E}$ & Plant & Both & USARPAC & Makua MR & None & In Preparation \\
\hline & Name & & & Both & USARPAC & Schofield Barracks MR & None & In Preparation \\
\hline $\begin{array}{l}\text { Diplazium } \\
\text { molokaiense }\end{array}$ & $\begin{array}{l}\text { No Common } \\
\text { Name }\end{array}$ & $E$ & Plant & Contig. & USARPAC & Schofield Barracks MR & None & In Preparation \\
\hline
\end{tabular}




\begin{tabular}{|c|c|c|c|c|c|c|c|c|}
\hline Scientific Name & Common Name & $\begin{array}{l}\text { Fed } \\
\text { Stat }\end{array}$ & Cat & Occur & MACOM & Installation & Crit & ESMP \\
\hline Dromus dromas & $\begin{array}{l}\text { Dromedary Pearly } \\
\text { Mussel }\end{array}$ & $E$ & Clam & Contig. & FORSCOM & Ft. Campbell & None & None \\
\hline $\begin{array}{l}\text { Drymarchon corais } \\
\text { couperi }\end{array}$ & $\begin{array}{l}\text { Eastern Indigo } \\
\text { Snake }\end{array}$ & $\mathrm{T}$ & Reptile & $\begin{array}{l}\text { Both } \\
\text { Both }\end{array}$ & $\begin{array}{l}\text { NGB } \\
\text { FORSCOM }\end{array}$ & $\begin{array}{l}\text { Camp Blanding TS } \\
\text { Ft. Stewart \& Hunter } \\
\text { Army Airfield }\end{array}$ & $\begin{array}{l}\text { None } \\
\text { None }\end{array}$ & $\begin{array}{r}\text { Completed } \\
\text { In Preparation }\end{array}$ \\
\hline Dubautia herbstobatae & Na`ena`e & $E$ & Plant & Both & USARPAC & Makua MR & None & In Preparation \\
\hline \multirow[t]{2}{*}{ Echinacea laevigata } & $\begin{array}{l}\text { Smooth } \\
\text { Coneflower }\end{array}$ & $E$ & Plant & Onsite & TRADOC & Ft. Jackson & None & In Preparation \\
\hline & & & & $\begin{array}{l}\text { Onsite } \\
\text { Contig. }\end{array}$ & $\begin{array}{l}\text { NGB } \\
\text { AMC }\end{array}$ & $\begin{array}{l}\text { Leesburg TS } \\
\text { Radford AAP }\end{array}$ & $\begin{array}{r}\text { None } \\
\text { NR }\end{array}$ & $\begin{array}{r}\text { In Preparation } \\
\text { None }\end{array}$ \\
\hline $\begin{array}{l}\text { Echinocactus } \\
\text { horizonthalonius } \\
\text { var. nicholii } \\
\end{array}$ & $\begin{array}{l}\text { Nichol's Turk's } \\
\text { Head Cactus }\end{array}$ & $E$ & Plant & Onsite & AMC & Yuma Proving Ground & None & None \\
\hline $\begin{array}{l}\text { Echinocereus fendleri } \\
\text { var. kuenzleri }\end{array}$ & $\begin{array}{l}\text { Kuenzler } \\
\text { Hedgehog Cactus }\end{array}$ & $E$ & Plant & Contig. & TRADOC & Ft. Bliss & None & None \\
\hline $\begin{array}{l}\text { Empidonax traillii } \\
\text { extimus }\end{array}$ & $\begin{array}{l}\text { Southwestern } \\
\text { Willow Flycatcher }\end{array}$ & $E$ & Bird & $\begin{array}{l}\text { Both } \\
\text { Both } \\
\text { Contig. } \\
\text { Contig. }\end{array}$ & $\begin{array}{l}\text { NGB } \\
\text { TRADOC } \\
\text { TRADOC } \\
\text { AMC }\end{array}$ & $\begin{array}{l}\text { Clark County TS } \\
\text { Ft. Bliss } \\
\text { Ft. Huachuca } \\
\text { White Sands Missile } \\
\text { Rng. }\end{array}$ & $\begin{array}{l}\text { None } \\
\text { None } \\
\text { None } \\
\text { None }\end{array}$ & $\begin{array}{r}\text { None } \\
\text { In Preparation } \\
\text { None } \\
\text { None }\end{array}$ \\
\hline Epicrates inornatus & Puerto Rican Boa & $E$ & Reptile & Onsite & FORSCOM & Ft. Buchanan & None & None \\
\hline Epioblasma walkeri & Tan Riffleshell & $\mathrm{E}$ & Clam & Contig. & FORSCOM & Ft. Campbell & None & None \\
\hline Eragrostis fosbergii & $\begin{array}{l}\text { Fosberg's Love } \\
\text { Grass }\end{array}$ & $\mathrm{PE}$ & Plant & Contig. & USARPAC & Schofield Barracks MR & NA & In Preparation \\
\hline Erigeron basalticus & Basalt Daisy & $\mathrm{C}$ & Plant & Both & FORSCOM & Yakima TC & NA & NA \\
\hline Erigeron lemmonii & $\begin{array}{l}\text { Lemmon } \\
\text { Fleabane }\end{array}$ & $\mathrm{C}$ & Plant & Onsite & TRADOC & Ft. Huachuca & NA & NA \\
\hline Etheostoma cragini & Arkansas Darter & $\mathrm{C}$ & Fish & $\begin{array}{l}\text { Contig. } \\
\text { Both }\end{array}$ & $\begin{array}{l}\text { NGB } \\
\text { FORSCOM }\end{array}$ & $\begin{array}{l}\text { Camp Crowder } \\
\text { Ft. Carson }\end{array}$ & $\begin{array}{l}\text { NA } \\
\text { NA }\end{array}$ & $\begin{array}{l}\text { NA } \\
\text { NA }\end{array}$ \\
\hline Etheostoma sellare & Maryland Darter & $E$ & Fish & Contig. & AMC & $\begin{array}{l}\text { Aberdeen Proving } \\
\text { Ground }\end{array}$ & None & None \\
\hline Eugenia koolauensis & Nioi & $E$ & Plant & $\begin{array}{l}\text { Onsite } \\
\text { Contig. }\end{array}$ & $\begin{array}{l}\text { USARPAC } \\
\text { USARPAC }\end{array}$ & $\begin{array}{l}\text { Kahuku TA } \\
\text { Kawailoa TA }\end{array}$ & $\begin{array}{l}\text { None } \\
\text { None }\end{array}$ & $\begin{array}{l}\text { In Preparation } \\
\text { In Preparation }\end{array}$ \\
\hline $\begin{array}{l}\text { Euphilotes enoptes } \\
\text { smithi }\end{array}$ & $\begin{array}{l}\text { Smith's Blue } \\
\text { Butterfly }\end{array}$ & $E$ & Insect & Both & TRADOC & Pom Annex & None & Completed \\
\hline $\begin{array}{l}\text { Euphorbia } \\
\text { haeleeleana }\end{array}$ & 'Akoko & PE & Plant & Onsite & USARPAC & Makua MR & NA & In Preparation \\
\hline $\begin{array}{l}\text { Falco femoralis } \\
\text { septentrionalis }\end{array}$ & $\begin{array}{l}\text { Northern } \\
\text { Aplomado } \\
\text { Falcon }\end{array}$ & $E$ & Bird & $\begin{array}{l}\text { Contig. } \\
\text { Both }\end{array}$ & $\begin{array}{l}\text { TRADOC } \\
\text { AMC }\end{array}$ & $\begin{array}{l}\text { Ft. Bliss } \\
\text { White Sands Missile } \\
\text { Rng. }\end{array}$ & $\begin{array}{l}\text { None } \\
\text { None }\end{array}$ & $\begin{array}{l}\text { In Preparation } \\
\text { In Preparation }\end{array}$ \\
\hline Falco peregrinus & Peregrine Falcon & $E(S / A)$ & Bird & Contig. & AMC & $\begin{array}{l}\text { Aberdeen Proving } \\
\text { Ground }\end{array}$ & None & None \\
\hline & & & & Both & NGB & Camp Navajo MR & None & NR \\
\hline & & & & Contig. & NGB & Camp Smith & None & None \\
\hline & & & & Both & USARC & Devens RFT.A & None & None \\
\hline & & & & Both & FORSCOM & Ft. Campbell & None & None \\
\hline & & & & Contig. & TRADOC & Ft. Eustis & None & In Preparation \\
\hline & & & & Both & FORSCOM & Ft. Hood & None & In Preparation \\
\hline & & & & Both & TRADOC & Ft. Huachuca & None & None \\
\hline & & & & Both & USARC & Ft. McCoy & None & None \\
\hline
\end{tabular}




\begin{tabular}{|c|c|c|c|c|c|c|c|c|}
\hline Scientific Name & Common Name & $\begin{array}{l}\text { Fed } \\
\text { Stat }\end{array}$ & Cat & Occur & MACOM & Installation & Crit & ESMP \\
\hline & & & & Both & FORSCOM & Ft. Riley & None & In Preparation \\
\hline & & & & Both & AMC & Sierra Army Depot & None & None \\
\hline & & & & Both & NGB & Stead TS & None & Completed \\
\hline & & & & Contig. & AMC & Sunflower AAP & None & None \\
\hline & & & & Contig. & MTMC & Sunny Point & None & None \\
\hline & & & & Contig. & FORSCOM & Yakima TC & None & NR \\
\hline \multirow{15}{*}{$\begin{array}{l}\text { Falco peregrinus } \\
\text { anatum }\end{array}$} & American & $E$ & Bird & Contig. & AMC & Dugway Proving & None & None \\
\hline & Peregrine & & & & & Ground & & \\
\hline & Falcon & & & Both & TRADOC & Ft. Bliss & None & In Preparation \\
\hline & & & & Both & FORSCOM & Ft. Carson & NR & NR \\
\hline & & & & Onsite & USARPAC & Ft. Greely & None & NR \\
\hline & & & & Both & USARC & Ft. Hunter Liggett & None & None \\
\hline & & & & Onsite & USARPAC & Ft. Richardson & None & NR \\
\hline & & & & Onsite & USARPAC & Ft. Wainwright & None & NR \\
\hline & & & & Both & AMC & Picatinny Arsenal & None & None \\
\hline & & & & & & Tacom-ARDEC & & \\
\hline & & & & Both & FORSCOM & Pinon Canyon MS & NR & NR \\
\hline & & & & Both & AMC & Redstone Arsenal & None & None \\
\hline & & & & Both & AMC & Tooele Army Depot & None & None \\
\hline & & & & Both & AMC & White Sands Missile & None & In Preparation \\
\hline & & & & & & Rng. & & \\
\hline Felis concolor coryi & Florida Panther & $\mathrm{E}$ & Mammal & NR & NGB & Camp Blanding TS & NR & NR \\
\hline Felis concolor cougar & Eastern Cougar & $\mathrm{E}$ & Mammal & Both & MTMC & Sunny Point & None & None \\
\hline \multirow{2}{*}{\multicolumn{2}{|c|}{ Flueggea neowawraea Mehamehame }} & $E$ & Plant & Onsite & USARPAC & Makua MR & None & In Preparation \\
\hline & & & & Contig. & USARPAC & Schofield Barracks MR & None & In Preparation \\
\hline \multirow[t]{2}{*}{ Fulica americana alai } & Hawaiian Coot & $E$ & Bird & Both & USARPAC & Dillingham TA & None & In Preparation \\
\hline & & & & Contig. & NGB & Kekaha LTA & None & NR \\
\hline \multirow{4}{*}{$\begin{array}{l}\text { Gallinula chloropus } \\
\text { sandvicensis }\end{array}$} & Hawaiian & $E$ & Bird & Onsite & NGB & Aahoaka \& Wailua & None & None \\
\hline & Gallinule & & & & & River LTA & & \\
\hline & & & & Both & USARPAC & Dillingham TA & None & In Preparation \\
\hline & & & & Contig. & NGB & Kekaha LTA & None & NR \\
\hline \multirow[t]{3}{*}{ Gardenia mannii } & Nanu & $\mathrm{PE}$ & Plant & Both & USARPAC & Kahuku TA & NA & In Preparation \\
\hline & & & & Both & USARPAC & Kawailoa TA & NA & In Preparation \\
\hline & & & & Both & USARPAC & Schofield Barracks MR & NA & In Preparation \\
\hline $\begin{array}{l}\text { Gilia tenuiflora } \\
\text { ssp. arenaria }\end{array}$ & $\begin{array}{l}\text { Monterey Sand } \\
\text { Gilia }\end{array}$ & $E$ & Plant & Both & TRADOC & Pom Annex & None & Completed \\
\hline \multirow[t]{2}{*}{ Gopherus agassizii } & Desert Tortoise & $\mathrm{T}$ & Reptile & Both & NGB & Clark County TS & None & None \\
\hline & & & & Both & FORSCOM & Ft. Irwin & Yes & In Preparation \\
\hline \multirow{3}{*}{$\begin{array}{l}\text { Gopherus agassizii } \\
\text { (Sonoran Population) }\end{array}$} & Sonoran & $\mathrm{T}(\mathrm{S} / \mathrm{A})$ & Reptile & Both & AMC & Yuma Proving Ground & None & None \\
\hline & Population of & & & & & & & \\
\hline & Desert Tortoise & & & & & & & \\
\hline \multicolumn{2}{|c|}{ Gopherus polyphemus Gopher Tortoise } & $\mathrm{T}$ & Reptile & Both & NGB & Camp Shelby & None & In Preparation \\
\hline Grus americana & Whooping Crane & $\mathrm{E}$ & Bird & Both & FORSCOM & Ft. Hood & None & In Preparation \\
\hline $\begin{array}{l}\text { Gymnogyps } \\
\text { californianus }\end{array}$ & California Condor & $E$ & Bird & Contig. & USARC & Ft. Hunter Liggett & None & None \\
\hline \multirow{6}{*}{$\begin{array}{l}\text { Haliaeetus } \\
\text { leucocephalus }\end{array}$} & Bald Eagle & $\mathrm{T}$ & Bird & Both & AMC & Aberdeen Proving & None & Completed \\
\hline & & & & & & Ground & & \\
\hline & & & & Onsite & AMC & Adelphi Army Research & None & Completed \\
\hline & & & & & & Labs & & \\
\hline & & & & Both & NGB & Atterbury Reserve & None & None \\
\hline & & & & & & Forces TA & & \\
\hline
\end{tabular}




\begin{tabular}{|c|c|c|c|c|c|c|c|}
\hline Scientific Name & Common Name & $\begin{array}{ll}\text { Fed } & \text { Cat } \\
\text { Stat } & \\
\end{array}$ & Occur & MACOM & Installation & Crit & ESMP \\
\hline & & & Both & NGB & Camp Blanding TS & None & Completed \\
\hline & & & Both & NGB & Camp Crowder & None & In Preparation \\
\hline & & & Both & NGB & Camp Grayling & None & Completed \\
\hline & & & Contig. & NGB & Camp Guernsey & None & None \\
\hline & & & Both & NGB & $\begin{array}{l}\text { Camp Joseph T. } \\
\text { Robinson }\end{array}$ & None & None \\
\hline & & & Both & NGB & Camp Murray & None & Completed \\
\hline & & & Both & NGB & Camp Navajo MR & None & In Preparation \\
\hline & & & Onsite & NGB & Camp Ripley & None & Completed \\
\hline & & & Both & NGB & Camp Roberts & None & None \\
\hline & & & Contig. & NGB & Camp Smith & None & None \\
\hline & & & Both & USARC & Devens RFT.A & None & None \\
\hline & & & Both & AMC & $\begin{array}{l}\text { Dugway Proving } \\
\text { Ground }\end{array}$ & None & None \\
\hline & & & Both & MDW & Ft. A.P. Hill & None & Completed \\
\hline & & & Both & MDW & Ft. Belvoir & None & In Preparation \\
\hline & & & Both & TRADOC & Ft. Benning & None & In Preparation \\
\hline & & & Both & TRADOC & Ft. Bliss & None & In Preparation \\
\hline & & & Both & FORSCOM & Ft. Campbell & None & None \\
\hline & & & Both & FORSCOM & Ft. Carson & NR & NR \\
\hline & & & Both & TRADOC & Ft. Eustis & None & In Preparation \\
\hline & & & Onsite & TRADOC & Ft. Gordon & None & In Preparation \\
\hline & & & Both & FORSCOM & Ft. Hood & None & In Preparation \\
\hline & & & Both & USARC & Ft. Hunter Liggett & None & In Preparation \\
\hline & & & Onsite & TRADOC & Ft. Jackson & None & None \\
\hline & & & Both & TRADOC & Ft. Knox & None & In Preparation \\
\hline & & & Both & TRADOC & Ft. Lee & None & In Preparation \\
\hline & & & Both & FORSCOM & Ft. Lewis & None & Completed \\
\hline & & & Both & USARC & Ft. McCoy & None & None \\
\hline & & & Both & USARC & Ft. Pickett & None & None \\
\hline & & & Both & FORSCOM & Ft. Riley & None & In Preparation \\
\hline & & & Contig. & TRADOC & Ft. Rucker & None & None \\
\hline & & & Both & FORSCOM & $\begin{array}{l}\text { Ft. Stewart \& Hunter } \\
\text { Army Airfield }\end{array}$ & None & In Preparation \\
\hline & & & Both & AMC & Holston AAP & None & In Preparation \\
\hline & & & Both & NGB & Macon TS & None & In Preparation \\
\hline & & & Both & AMC & $\begin{array}{l}\text { Newport Chemical } \\
\text { Depot }\end{array}$ & None & None \\
\hline & & & Both & AMC & $\begin{array}{l}\text { Picatinny Arsenal } \\
\text { Tacom-ARDEC }\end{array}$ & None & None \\
\hline & & & Both & AMC & Pine Bluff Arsenal & None & None \\
\hline & & & Both & FORSCOM & Pinon Canyon MS & NR & NR \\
\hline & & & Contig. & AMC & Ravenna AAP & None & None \\
\hline & & & Both & AMC & Redstone Arsenal & None & None \\
\hline & & & Both & AMC & Rock Island Arsenal & None & None \\
\hline & & & Both & AMC & Savanna Army Depot & None & Completed \\
\hline & & & Both & AMC & Sierra Army Depot & None & None \\
\hline & & & Both & NGB & Skelton TS & None & In Preparation \\
\hline & & & Both & NGB & Stead TS & None & Completed \\
\hline & & & Both & AMC & Sunflower AAP & None & None \\
\hline & & & Both & MTMC & Sunny Point & None & None \\
\hline
\end{tabular}




\begin{tabular}{|c|c|c|c|c|c|c|c|c|}
\hline Scientific Name & Common Name & $\begin{array}{l}\text { Fed } \\
\text { Stat }\end{array}$ & Cat & Occur & MACOM & Installation & Crit & ESMP \\
\hline & & & & Both & AMC & Tooele Army Depot & None & None \\
\hline & & & & Both & NGB & Truman TS & None & In Preparation \\
\hline & & & & Both & AMC & Twin Cities AAP & None & None \\
\hline & & & & Contig. & NGB & Wappapello TS & None & None \\
\hline & & & & Both & USMA & West Point & None & In Preparation \\
\hline & & & & Both & AMC & $\begin{array}{l}\text { White Sands Missile } \\
\text { Rng. }\end{array}$ & None & None \\
\hline & & & & Both & FORSCOM & Yakima TC & None & None \\
\hline Haplostachys & No Common & $E$ & Plant & Onsite & NGB & Keamuku LTA & None & None \\
\hline haplostachya & Name & & & Both & USARPAC & Pohakuloa TA & None & In Preparation \\
\hline \multirow[t]{3}{*}{ Hedeoma todsenii } & Todsen's & $E$ & Plant & Contig. & TRADOC & Ft. Bliss & None & None \\
\hline & Pennyroyal & & & Both & AMC & White Sands Missile & None & None \\
\hline & & & & & & Rng. & & \\
\hline Hedyotis coriacea & Kio'ele & $\mathrm{E}$ & Plant & Onsite & USARPAC & Pohakuloa TA & None & In Preparation \\
\hline \multirow[t]{2}{*}{ Hedyotis parvula } & No Common & $E$ & Plant & Both & USARPAC & Makua MR & None & In Preparation \\
\hline & Name & & & & & & & \\
\hline \multirow[t]{2}{*}{ Helianthus eggertii } & Eggert's & PT & Plant & Both & NGB & Tullahoma TS & NA & In Preparation \\
\hline & Sunflower & & & & & & & \\
\hline Helonias bullata & Swamp Pink & $\mathrm{T}$ & Plant & Onsite & MDW & Ft. A.P. Hill & None & None \\
\hline Hemignathus munroi & $\begin{array}{l}\text { ‘kiapola`au } \\
\text { (Honeycreeper) }\end{array}$ & $E$ & Bird & Both & USARPAC & Pohakuloa TA & None & In Preparation \\
\hline \multirow{3}{*}{$\begin{array}{l}\text { Hesperomannia } \\
\text { arborescens }\end{array}$} & No Common & $E$ & Plant & Both & USARPAC & Kahuku TA & None & In Preparation \\
\hline & Name & & & Both & USARPAC & Kawailoa TA & None & In Preparation \\
\hline & & & & Both & USARPAC & Schofield Barracks MR & None & In Preparation \\
\hline \multirow{3}{*}{\multicolumn{9}{|c|}{$\begin{array}{l}\text { Hibiscus } \\
\text { brackenridgei ssp. } \\
\text { mokuleianus }\end{array}$}} \\
\hline & & & & & & & & \\
\hline & & & & & & & & \\
\hline Himantopus & Hawaiian Stilt & $E$ & Bird & Both & USARPAC & Dillingham TA & None & In Preparation \\
\hline mexicanus & & & & Contig. & NGB & Kekaha LTA & None & NR \\
\hline \multicolumn{9}{|l|}{ knudseni } \\
\hline Howellia aquatilis & Water Howellia & $\mathrm{T}$ & Plant & Both & FORSCOM & Ft. Lewis & None & In Preparation \\
\hline \multirow[t]{2}{*}{ Hymenoxys texana } & Texas Prairie & $E$ & Plant & Both & NGB & Addicks Reservoir & None & NR \\
\hline & Dawn-flower & & & & & & & \\
\hline Isodendrion hosakae & Aupaka & $\mathrm{E}$ & Plant & Onsite & NGB & Keamuku LTA & None & None \\
\hline Isodendrion laurifolium & Aupaka & $\mathrm{PE}$ & Plant & Both & USARPAC & Schofield Barracks MR & NA & In Preparation \\
\hline \multirow[t]{2}{*}{ Isoetes louisianensis } & Lousiana & $E$ & Plant & Both & NGB & Camp Shelby & None & None \\
\hline & Quillwort & & & & & & & \\
\hline \multirow[t]{3}{*}{ Isotria medeoloides } & Small Whorled & $\mathrm{T}$ & Plant & Onsite & MDW & Ft. A.P. Hill & None & None \\
\hline & Pogonia & & & Contig. & NGB & Regional Training & None & None \\
\hline & & & & & & Institute & & \\
\hline \multirow[t]{2}{*}{ Labordia cyrtandrae } & Kamakahala & PE & Plant & Both & USARPAC & Kawailoa TA & NA & In Preparation \\
\hline & & & & Onsite & USARPAC & Schofield Barracks MR & NA & In Preparation \\
\hline \multirow[t]{3}{*}{ Lampsilis abrupta } & Pink Mucket & $E$ & Clam & Contig. & FORSCOM & Ft. Campbell & None & None \\
\hline & Pearlymussel & & & Contig. & NGB & Skelton TS & None & In Preparation \\
\hline & & & & Contig. & NGB & Wappapello TS & None & None \\
\hline \multirow[t]{2}{*}{ Lampsilis higginsi } & Higgins' Eye & $E$ & Clam & Both & AMC & Savanna Army Depot & None & None \\
\hline & Pearlymussel & & & & & & & \\
\hline \multirow{3}{*}{$\begin{array}{l}\text { Lasiurus cinereus } \\
\text { semotus }\end{array}$} & Hawaiian Hoary & $E$ & Mammal & Both & NGB & Aahoaka \& Wailua & None & None \\
\hline & Bat & & & & & River LTA & & \\
\hline & & & & Both & USARPAC & Kawailoa TA & None & In Preparation \\
\hline
\end{tabular}




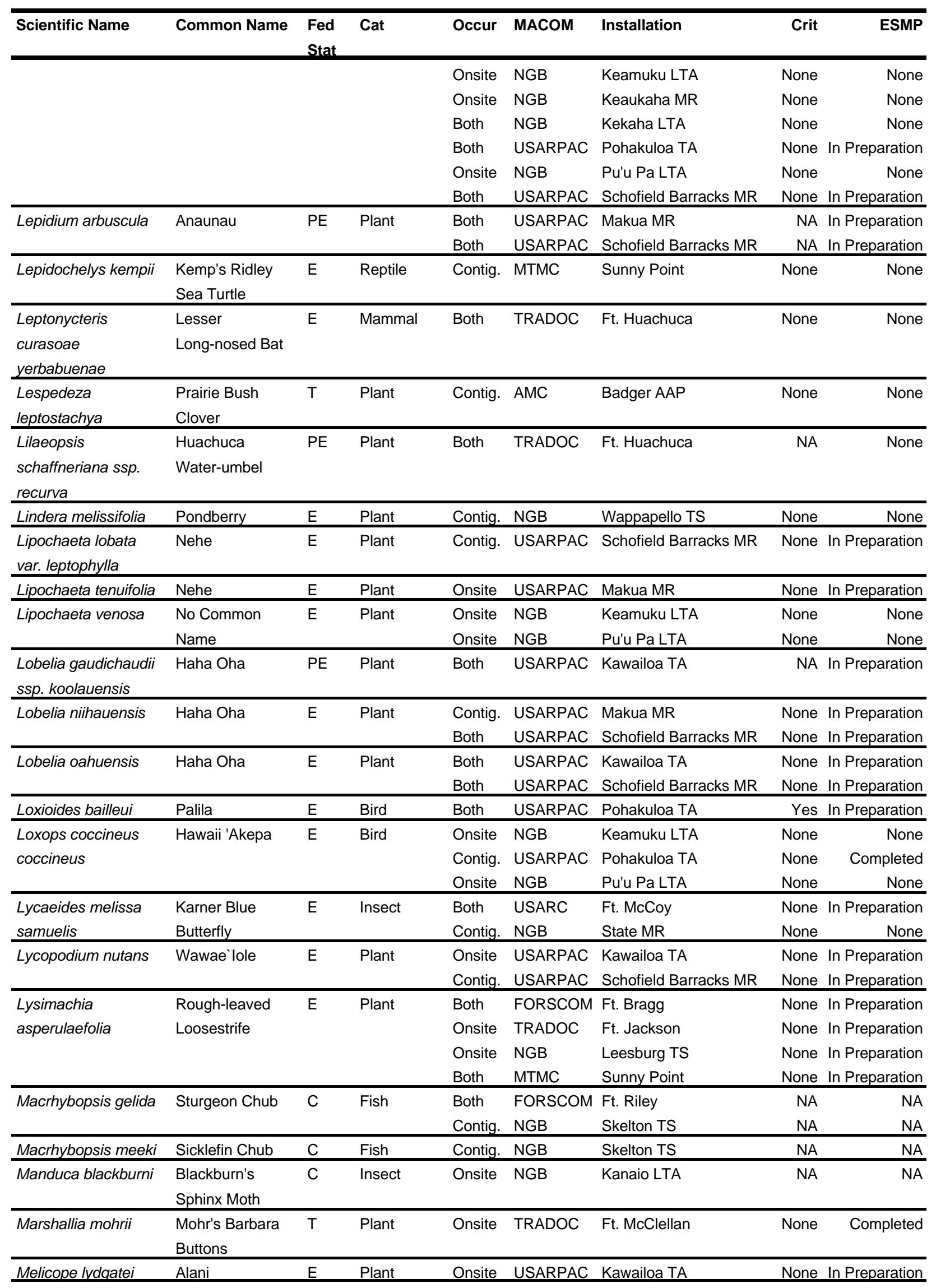




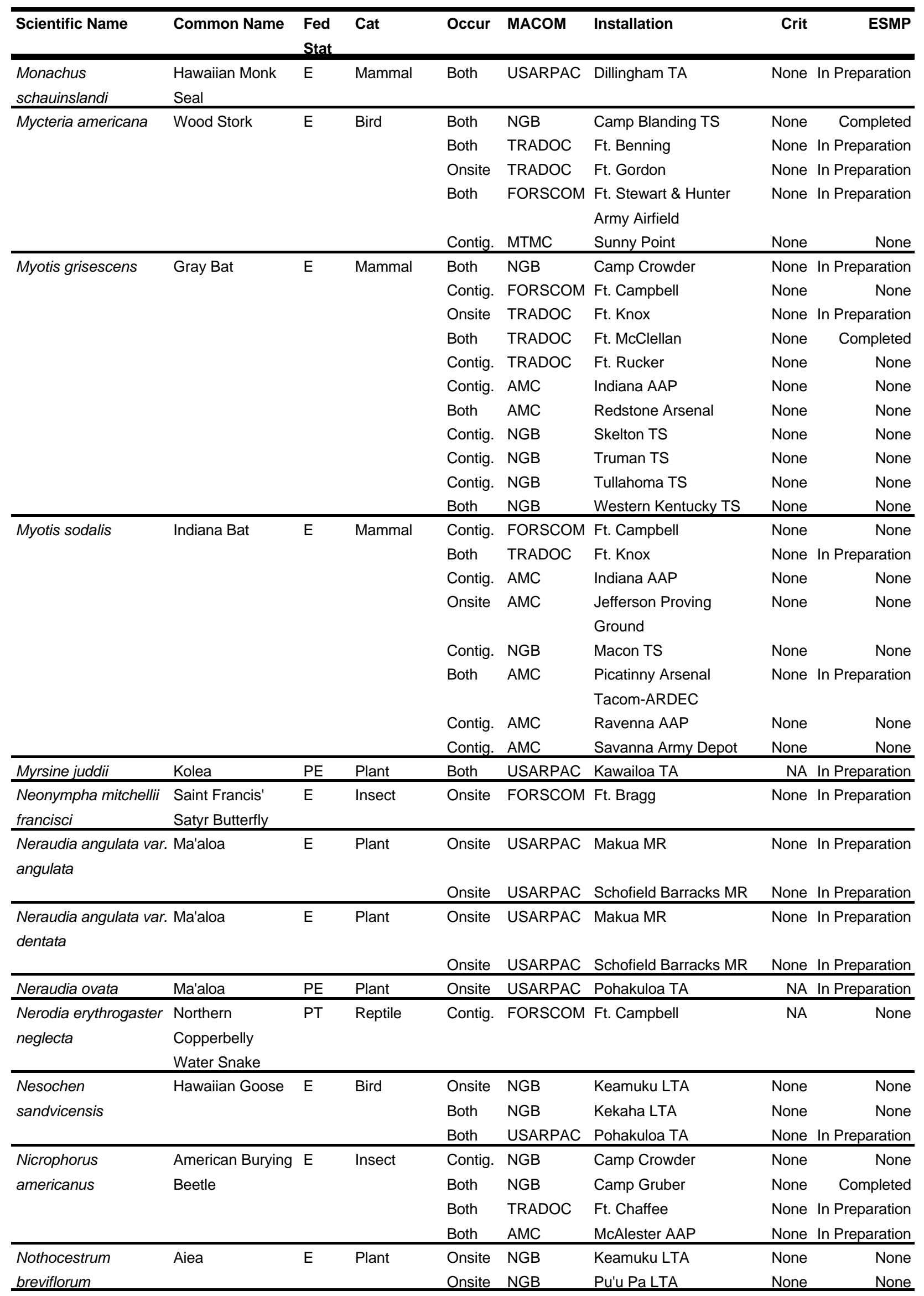




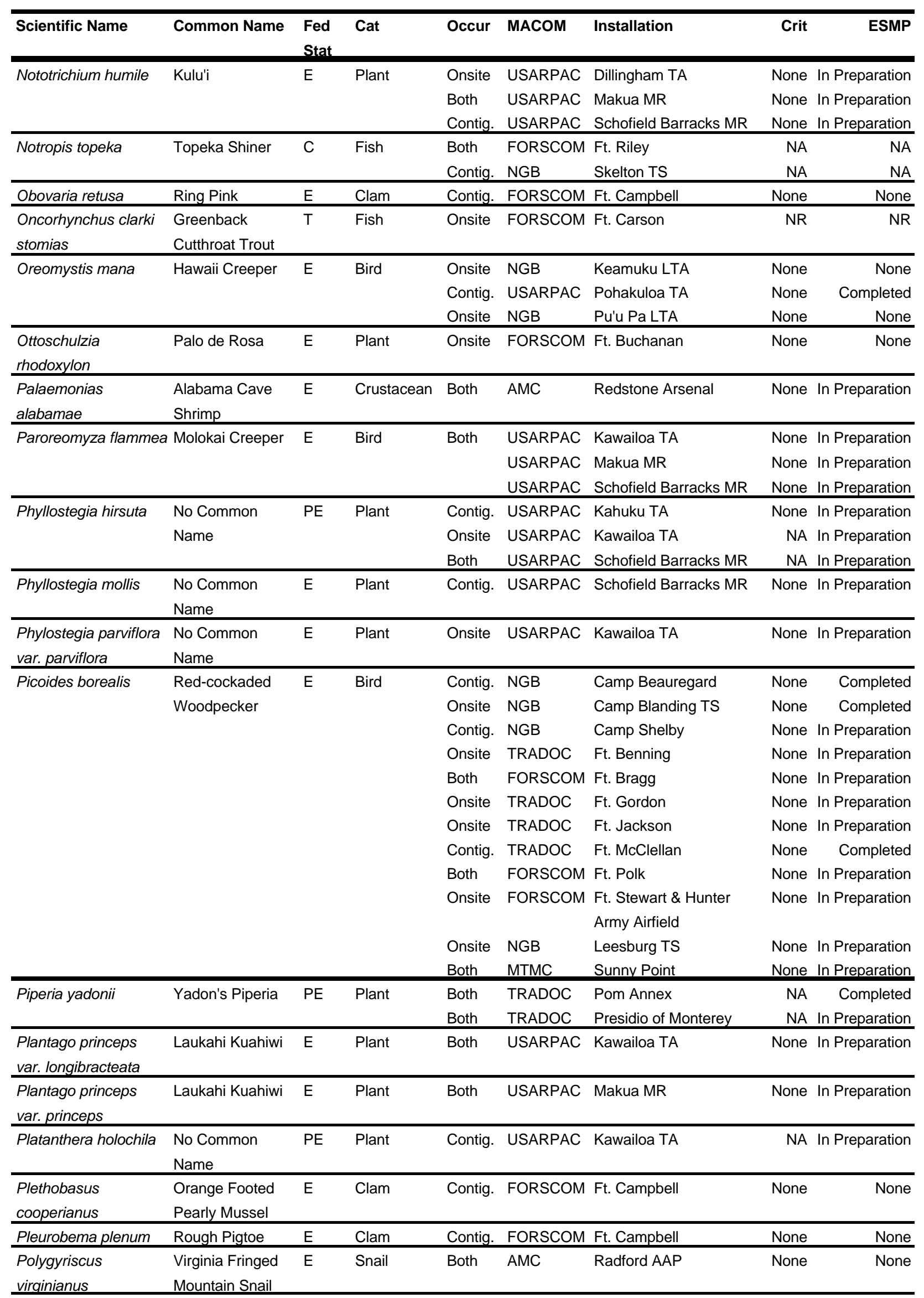




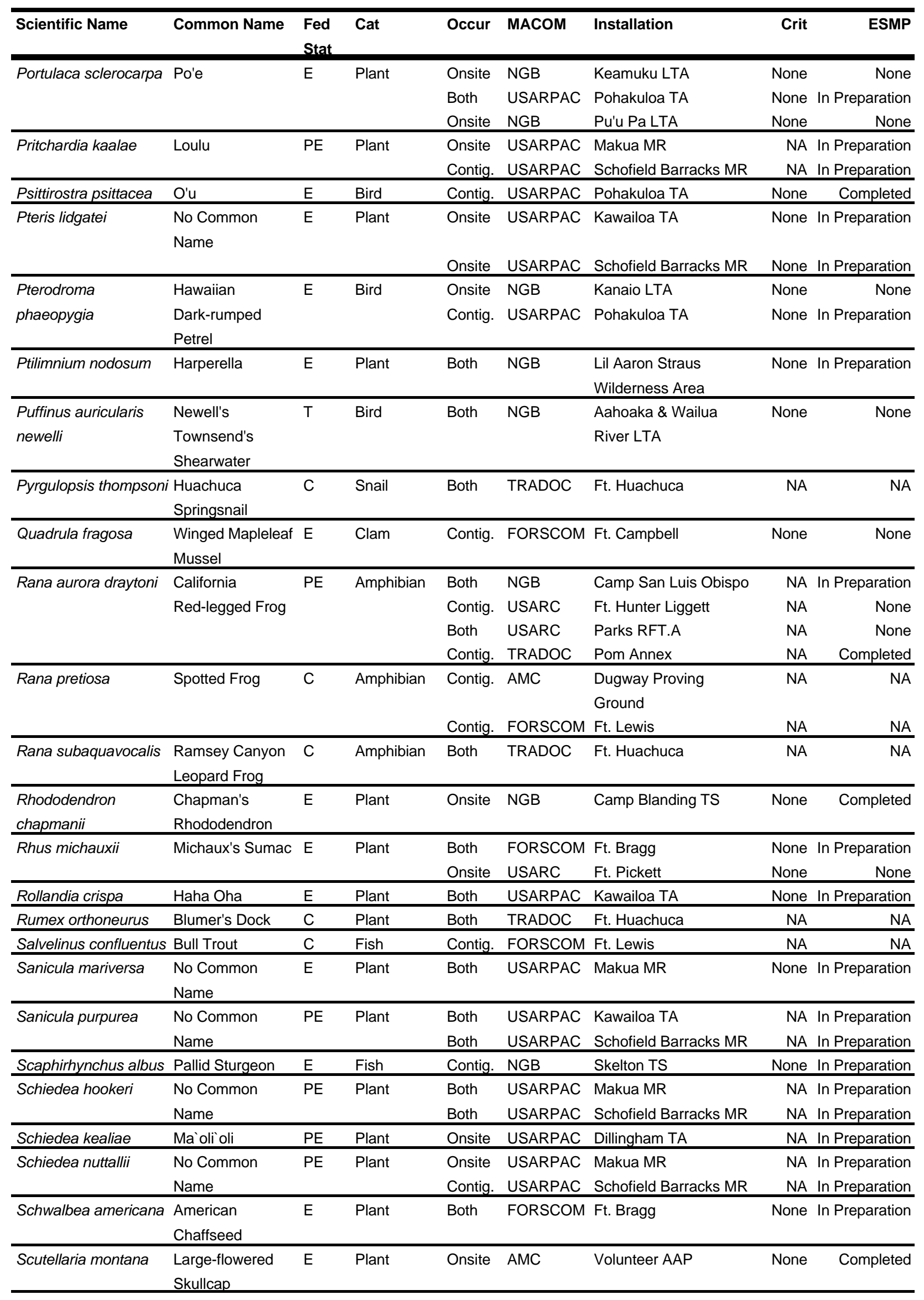




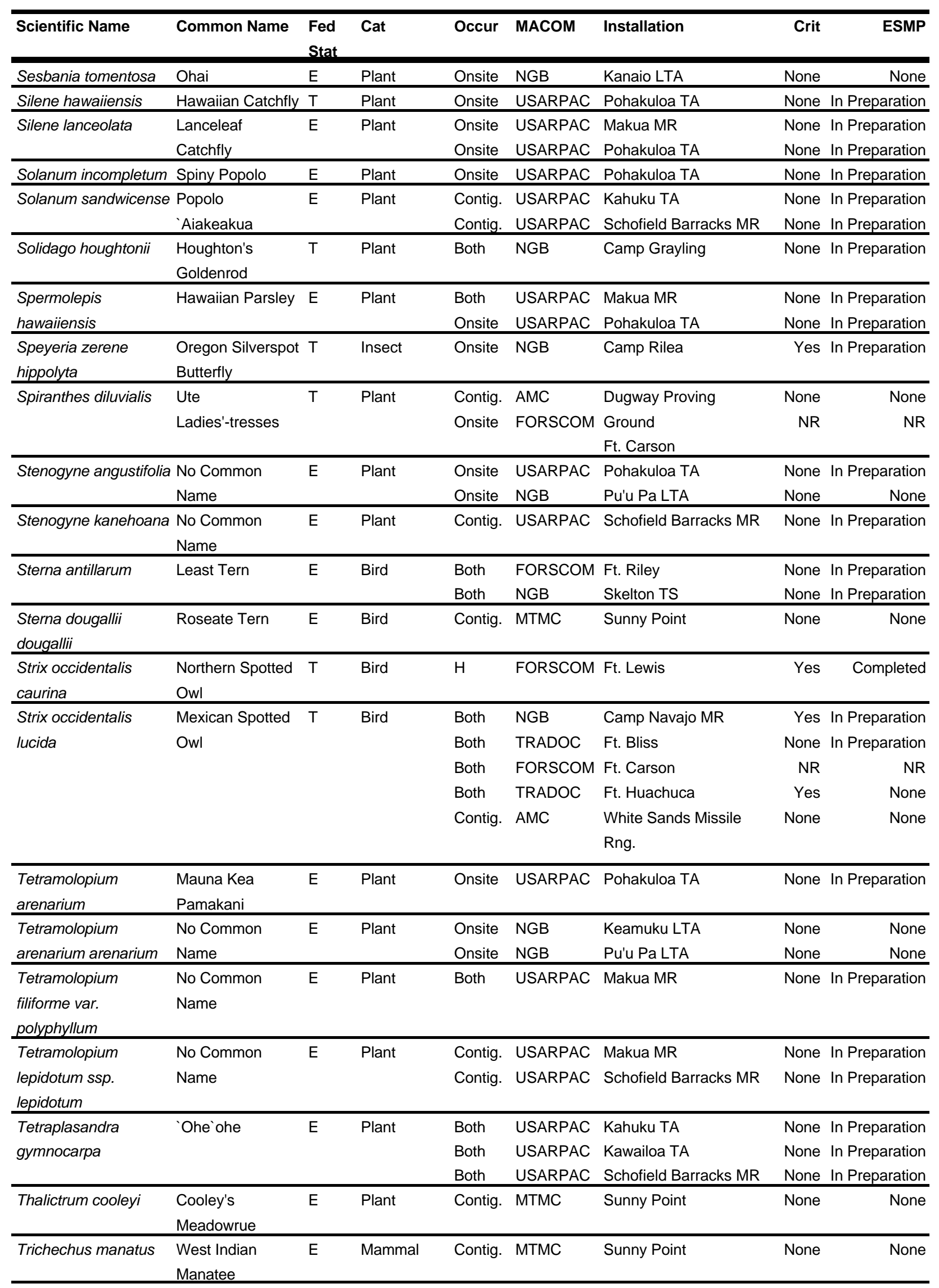




\begin{tabular}{|c|c|c|c|c|c|c|c|c|}
\hline Scientific Name & Common Name & $\begin{array}{l}\text { Fed } \\
\text { Stat }\end{array}$ & Cat & Occur & MACOM & Installation & Crit & ESMP \\
\hline Trifolium stoloniferum & $\begin{array}{l}\text { Running Buffalo } \\
\text { Clover }\end{array}$ & $E$ & Plant & Both & AMC & Bluegrass Army Depot & None & None \\
\hline Trillium reliquum & Relict Trillium & $\mathrm{E}$ & Plant & Onsite & TRADOC & Ft. Benning & None & In Preparation \\
\hline Urera kaalae & Opuhe & $\mathrm{E}$ & Plant & Both & USARPAC & Schofield Barracks MR & None & In Preparation \\
\hline $\begin{array}{l}\text { Ursus americanus } \\
\text { floridanus }\end{array}$ & Florida Black Bear & C & Mammal & Both & NGB & Camp Blanding TS & NA & NA \\
\hline Vigna o-wahuensis & $\begin{array}{l}\text { No Common } \\
\text { Name }\end{array}$ & $E$ & Plant & Onsite & NGB & Keamuku LTA & None & None \\
\hline $\begin{array}{l}\text { Viola chamissoniana } \\
\text { ssp. }\end{array}$ & Pamakani & $E$ & Plant & $\begin{array}{l}\text { Onsite } \\
\text { Both }\end{array}$ & $\begin{array}{l}\text { USARPAC } \\
\text { USARPAC } \\
\end{array}$ & $\begin{array}{l}\text { Makua MR } \\
\text { Schofield Barracks MR }\end{array}$ & $\begin{array}{l}\text { None } \\
\text { None } \\
\end{array}$ & $\begin{array}{l}\text { In Preparation } \\
\text { In Preparation } \\
\end{array}$ \\
\hline Viola oahuensis & $\begin{array}{l}\text { No Common } \\
\text { Name }\end{array}$ & PE & Plant & $\begin{array}{l}\text { Both } \\
\text { Both }\end{array}$ & $\begin{array}{l}\text { USARPAC } \\
\text { USARPAC }\end{array}$ & $\begin{array}{l}\text { Kawailoa TA } \\
\text { Schofield Barracks MR }\end{array}$ & $\begin{array}{l}\mathrm{NA} \\
\mathrm{NA}\end{array}$ & $\begin{array}{l}\text { In Preparation } \\
\text { In Preparation }\end{array}$ \\
\hline Vireo atricapillus & $\begin{array}{l}\text { Black-capped } \\
\text { Vireo }\end{array}$ & $E$ & Bird & $\begin{array}{l}\text { Both } \\
\text { Both } \\
\text { Both } \\
\text { Both } \\
\end{array}$ & $\begin{array}{l}\text { NGB } \\
\text { FORSCOM } \\
\text { MEDCOM } \\
\text { TRADOC } \\
\end{array}$ & $\begin{array}{l}\text { Camp Barkeley } \\
\text { Ft. Hood } \\
\text { Ft. Sam Houston } \\
\text { Ft. Sill }\end{array}$ & $\begin{array}{l}\text { None } \\
\text { None } \\
\text { None } \\
\text { None } \\
\end{array}$ & $\begin{array}{r}\text { Completed } \\
\text { In Preparation } \\
\text { In Preparation } \\
\text { In Preparation } \\
\end{array}$ \\
\hline Vireo bellii pusillus & Least Bell's Vireo & $\mathrm{E}$ & Bird & $\begin{array}{l}\text { Onsite } \\
\text { Both } \\
\end{array}$ & $\begin{array}{l}\text { NGB } \\
\text { USARC }\end{array}$ & $\begin{array}{l}\text { Camp San Luis Obispo } \\
\text { Ft. Hunter Liggett }\end{array}$ & $\begin{array}{l}\text { None } \\
\text { None } \\
\end{array}$ & $\begin{array}{r}\text { In Preparation } \\
\text { None } \\
\end{array}$ \\
\hline $\begin{array}{l}\text { Vulpes macrotis } \\
\text { mutica }\end{array}$ & $\begin{array}{l}\text { San Joaquin Kit } \\
\text { Fox }\end{array}$ & $E$ & Mammal & $\begin{array}{l}\text { Both } \\
\text { Both } \\
\text { Contig. }\end{array}$ & $\begin{array}{l}\text { NGB } \\
\text { USARC } \\
\text { USARC }\end{array}$ & $\begin{array}{l}\text { Camp Roberts } \\
\text { Ft. Hunter Liggett } \\
\text { Parks RFT.A }\end{array}$ & $\begin{array}{l}\text { None } \\
\text { None } \\
\text { None }\end{array}$ & $\begin{array}{r}\text { None } \\
\text { In Preparation } \\
\text { None }\end{array}$ \\
\hline Vulpes velox & Swift Fox & $\mathrm{C}$ & Mammal & Both & FORSCOM & Pinon Canyon MS & NA & NA \\
\hline Xyris tennesseensis & $\begin{array}{l}\text { Tennessee } \\
\text { Yellow-eyed } \\
\text { Grass } \\
\end{array}$ & $E$ & Plant & $\begin{array}{l}\text { Both } \\
\text { Both }\end{array}$ & $\begin{array}{l}\text { AMC } \\
\text { TRADOC }\end{array}$ & $\begin{array}{l}\text { Anniston Army Depot } \\
\text { Ft. McClellan }\end{array}$ & $\begin{array}{l}\text { None } \\
\text { None }\end{array}$ & $\begin{array}{l}\text { Completed } \\
\text { Completed }\end{array}$ \\
\hline $\begin{array}{l}\text { Zanthoxylum } \\
\text { hawaiiense }\end{array}$ & $A^{\prime} e$ & $\mathrm{E}$ & Plant & $\begin{array}{l}\text { Onsite } \\
\text { Both }\end{array}$ & $\begin{array}{l}\text { NGB } \\
\text { USABPAC }\end{array}$ & $\begin{array}{l}\text { Keamuku LTA } \\
\text { Pohakuloa TA }\end{array}$ & $\begin{array}{l}\text { None } \\
\text { None }\end{array}$ & $\begin{array}{r}\text { None } \\
\text { In Prebaration }\end{array}$ \\
\hline
\end{tabular}


USACERL DISTRIBUTION

Chief of Engineers

ATTN: CEHEC-IM-LH (2)

ATTN: CEHEC-IM-LP (2)

ATTN: CECC-R

ATTN: CERD-L

ATTN: CERD-M

Defense Tech Info Center 22304

ATTN: DTIC-O (2)

HQDA,

ATTN: DAJA-EL

HQ TRADOC

ATTN: ATBO-FN

ATTN: ATBO-SE

US AEC

ATTN: SFIM-AEC-ECN (200)

ATTN: SFIM-AEC-ECM (2)

ATTN: SFIM-AEC-EC

HQ FORSCOM

ATTN: AFPI-ENE

ATTN: AFOP-TE

ODUSD (ES) C

AMC

ATTN: AMXEN-M

ATTN: AMXEN-U

US Military Academy

ATTN: MAEN-ED

ATCS

ATTN: ATIC-CTS

DA-Env Programs

ATTN: NGB-ILE-EC

ATTN: NGB-ARE-C

US Army Medical Command

ATTN: MCFA-E

SERDP Program Office

Arlington, VA

HQ US Army Pacific

ATTN: APEN-E
Asst Sec of the Army,

(Installations, Logistics, and Environment)

Military Dist of Washington

ATTN: ANEN-ES (2)

US Fish \& Wildlife Service ATTN: ARLSQ 452

HQ AF Center for Env Excellence ATTN: AFCEE/ECR

HQ DA

ATTN: DAMO-TRO

ATTN: DAMO-TRS

Mil Traffic Management Command ATTN: MTPAL-FE

USARCOM ATTN: AFRC-ENP-NC ATTN: AFRC-ENS-E

US Army Engineering \& Spt Ctr ATTN: CEHND-ED-S

US Army Logistics Management ATTN: ATSZ-LSE

Commander, Fort McCoy ATTN: AFRC-FM

Defense Environmental Security Corp

HQDA ACSIM

ATTN: DAIM-ED-R (5)

ATTN: DAIM-ED

US Army Space \& Strategic Cmd ATTN: CSSD-EN-V 